\title{
Proteoglycan IMPG2 Shapes the Interphotoreceptor Matrix and Modulates Vision
}

\author{
${ }^{\circ}$ Ezequiel M. Salido and ${ }^{\circledR}$ Visvanathan Ramamurthy \\ Department of Biochemistry, Ophthalmology and Visual Sciences, West Virginia University, Morgantown, West Virginia 26506
}

Photoreceptor neurons are surrounded by an extracellular matrix, called the interphotoreceptor matrix (IPM). Activities crucial to vision occur within the IPM, including trafficking of nutrients and metabolites, retinal attachment, and interactions needed for normal outer segment phagocytosis. The IPM includes the following two unique proteoglycans: IPM proteoglycan 1 (IMPG1) and IMPG2. Patients with mutations in IMPG1/IMPG2 develop visual deficits with subretinal material accumulation, highlighting the critical role of the IPM in vision. To determine the role of these proteoglycans in retinal physiology and the pathologic mechanisms that lead to vision loss, we generated mouse models lacking IMPG1/IMPG2. In normal retina, IMPG1 and IMPG2 occupy distinct IPM compartments, represent the main source of chondroitin sulfate and are fundamental for the constitution of the cone-specific glycocalyx stained by the PNA (peanut agglutinin) lectin marker. No evident morphologic or functional deficits were found in mice lacking IMPG1. In the absence of IMPG2, IMPG1 abnormally accumulated at the subretinal space need, likely leading to the formation of subretinal lesions and reduced visual function. Interestingly, mice lacking both IMPG1 and IMPG2, regardless of sex, showed normal retinal structure and function, demonstrating that the aberrant IMPG1 distribution is the main cause of the visual alterations observed in the absence of IMPG2. In conclusion, our results show the dependence of secreted proteoglycans such as IMPG1 on the extracellular environment to properly integrate into the matrix, demonstrate the role of IMPG2 in shaping the IPM, and shed light on the potential mechanisms leading to the development of subretinal lesions and vision loss.

Key words: age-related macular degeneration; blindness; chondroitin sulfate; extracellular matrix; retina; vitelliform macular dystrophy

Significance Statement

The photoreceptors are specialized neurons that drive phototransduction in the mammalian retina. These cells are organized and surrounded by an extracellular matrix, the interphotoreceptor matrix (IPM). Mutations in IPM proteoglycans are associated with blindness in humans. Our studies show that two specific proteoglycans of the IPM, IPM proteoglycan 1 (IMPG1) and IMPG2, form a dynamic structure with distinct localization and dependency. When IMPG2 is absent, IMPG1 cannot integrate into the IPM, leading to abnormal proteoglycan accumulation and visual deficits. This work adds a new layer of understanding to IPM physiology and describes the pathologic events following deficits in proteoglycans, providing novel possibilities for visual restoration in patients with IMPG-related pathologies.

Received Dec. 4, 2019; revised Jan. 29, 2020; accepted Mar. 19, 2020

The authors declare no competing financial interests.

Author contributions: E.M.S. and V.R. designed research; E.M.S. performed research; E.M.S. and V.R. analyzed data; E.M.S. and V.R. wrote the paper.

This work was supported by Knight Templar Eye Foundation (E.M.S.); National Institutes of Health Grants R01-EY-028035 (V.R.), R01-EY-025536 (V.R.), and R21-EY-027707 (V.R.); and West Virginia Lions and Lions Club International Foundation. We thank Thamaraiselvi Saravanan and Angelica Jacques for support in the maintenance of animal models and genotyping, Lucia Bonifacini for aiding in the preparation of figures, Scott Rhodes for help editing this article, and Peter Mathers and the transgenic core in generation of the animal models.

Correspondence should be addressed to Ezequiel M. Salido at ezequielsalido@gmail.com or Visvanathan Ramamurthy at ramamurthyv@mix.wvu.edu.

https://doi.org/10.1523/JNEUROSCI.2994-19.2020

Copyright $(2020$ the authors

\section{Introduction}

The extracellular matrx (ECM) in the CNS is an organized extracellular structure synthesized by molecules secreted by neurons and glial cells. ECMs play fundamental roles in cortical circuit development and plasticity (Berardi et al., 2004; Hensch, 2005). Deficits in the ECM are associated with epilepsy, autism, schizophrenia, Alzheimer's disease, and blindness (Xie et al., 2013; Arranz et al., 2014; Ishikawa et al., 2015; Sorg et al., 2016; Nicholson and Hrabětová, 2017; Wen et al., 2018). The ECM surrounding the photoreceptor outer segment (OS) and inner segment (IS) in the retina is called the interphotoreceptor matrix (IPM), and it extends from the Müller cell apical microvilli to the apical retinal pigment epithelium (RPE) microvilli (Ishikawa et al., 2015). 
A IMPG1

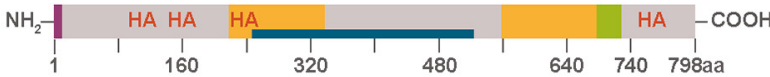

IMPG2

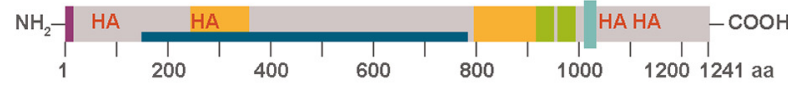

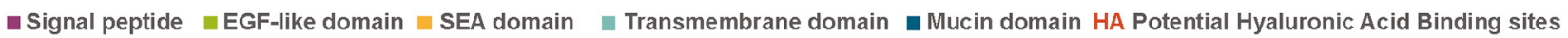
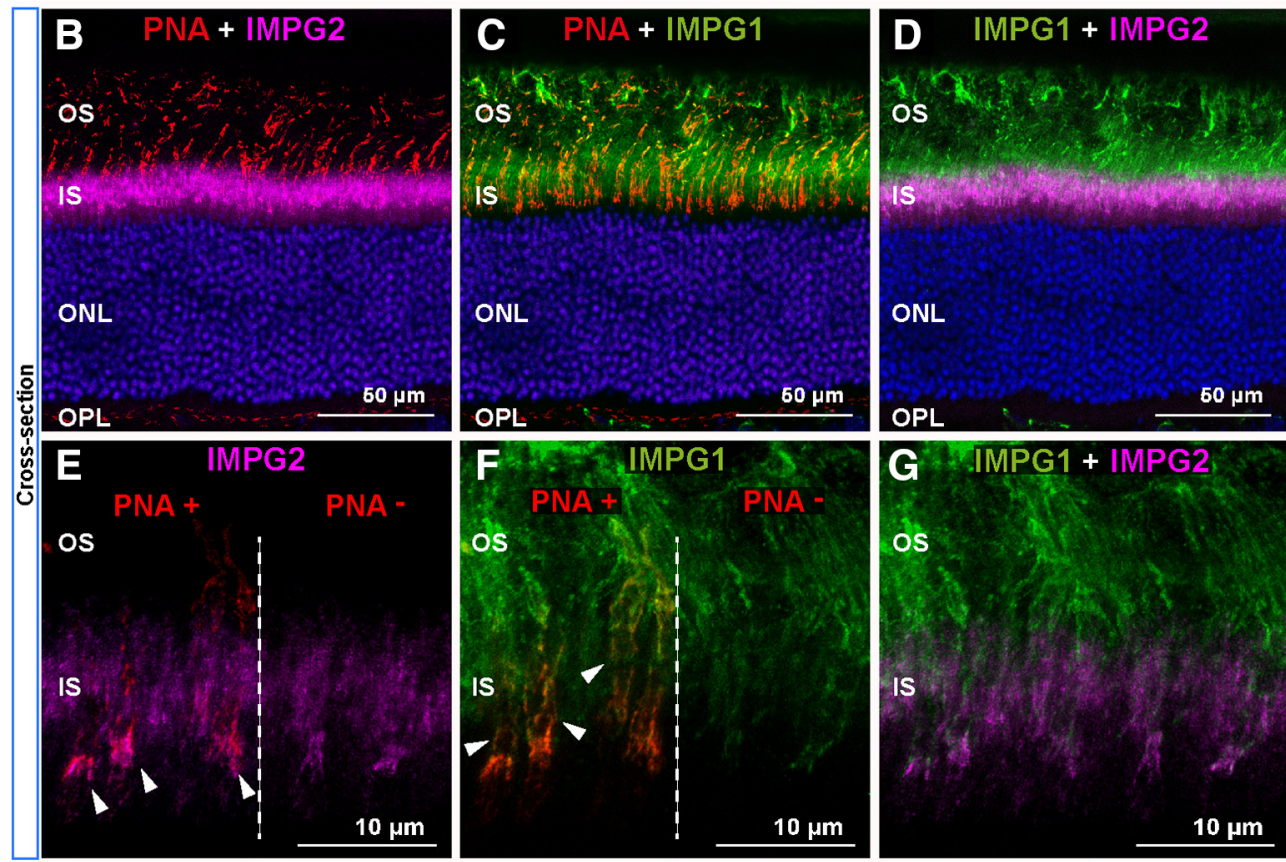

K

| PNA IIMPG1 \% IMPG2
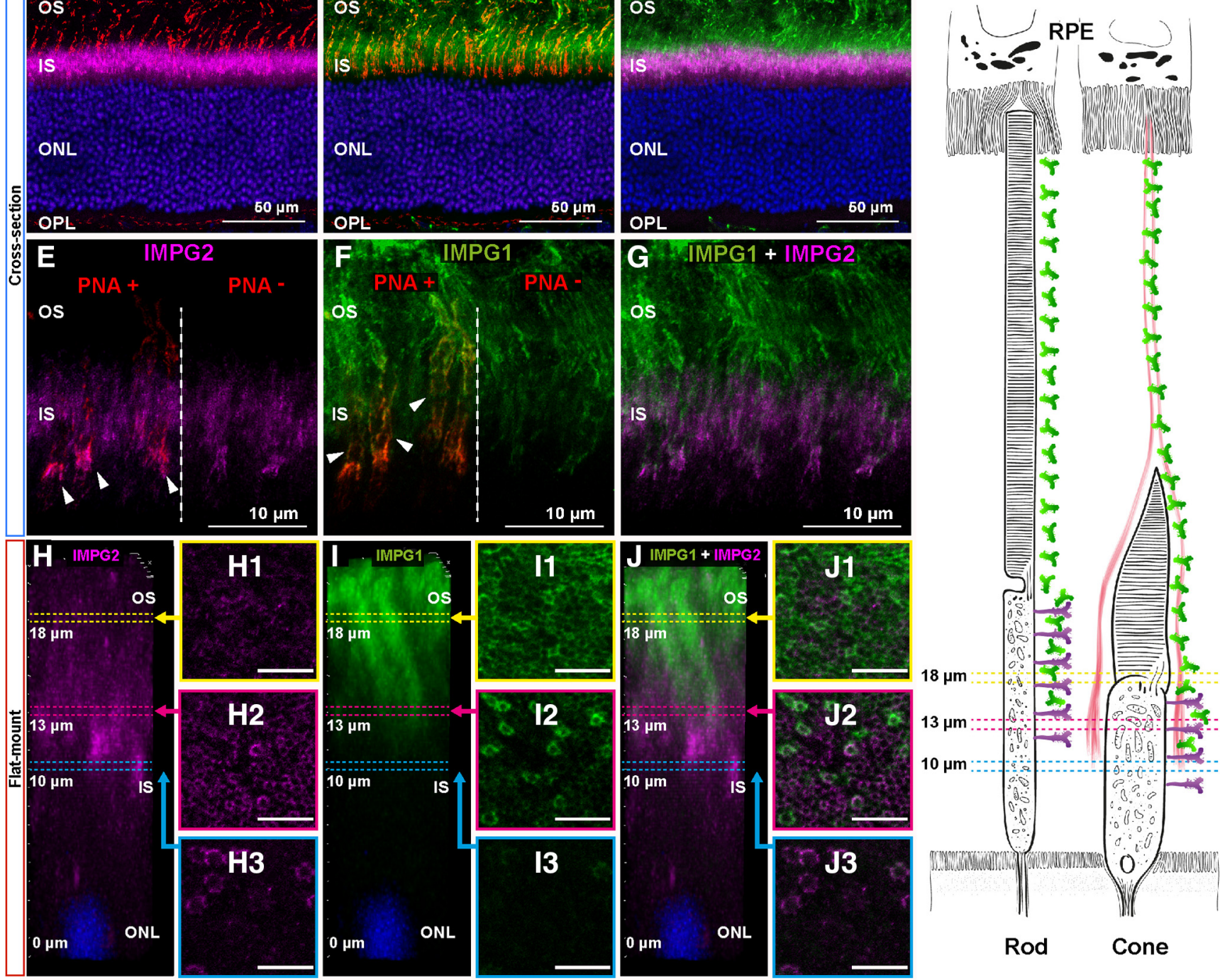

Rod

Cone

Figure 1. Localization of IMPG1 and IMPG2 proteoglycans in the IPM. Immunohistochemistry using retinas from wild-type mice with IMPG1 (green) and IMPG2 (magenta) antibodies and cone-specific lectin PNA (red). A, Predicted structural domains of IMPG1 and IMPG2 proteins. Both IMPG1 and IMPG2 proteins have abundant 0 - and N-glycosylation sites as well as potential hyaluronic acid attachment sites. SEA, Sperm protein, enterokinase, agrin domain; EGF, epidermal growth factor. $\boldsymbol{B}-\boldsymbol{D}$, Retinal cross-sections from 45 -days-0ld animals imaged at low magnification. $\boldsymbol{E}-\boldsymbol{G}$, Retinal cross sections imaged at higher magnification. Arrowheads indicate colocalization of PNA with IMPG2 and IMPG1 in $\boldsymbol{E}$ and $\boldsymbol{F}$, respectively. $\boldsymbol{H}-\boldsymbol{J}$, Flat-mount $3 \mathrm{D}$ reconstruction of the retina focused on a single cone. On the right side of the $3 \mathrm{D}$ reconstruction image, raw confocal images of the flat-mounted retina used for $3 \mathrm{D}$ reconstruction selected at three different heights from the ONL $(10,13$, and $18 \mu \mathrm{m})$. H.3, I.3, There is a robust cone-IMPG2 stain at $10 \mu \mathrm{m}$ above the ONL (H.3), and a weak IMPG1 mark (I.3). H.2, I.2, J.2, At 13 $\mu \mathrm{m}$, rods and cones stain with IMPG2 and IMPG1. J.2 shows IMPG1 from cones in green, surrounded by a smaller reticulated magenta pattern that belongs to rod-IMPG2 staining. H.1, I.1, At 18 $\mu \mathrm{m}$ height, IMPG2 stain is faint and unstructured (H.1), while IMPG1 is strong with a uniform reticular pattern (I.1). Scale bars, $\boldsymbol{H}-\boldsymbol{J}, 10 \mu \mathrm{m}$. K, Schematic representation of IMPG1, IMPG2, and PNA in cones and rods, combined with a height reference of the sections shown in subfigures $\boldsymbol{H}, \boldsymbol{I}$, and $\boldsymbol{J}$. Pictures are representative of three independent experiments.

The IPM constitutes the central transit zone for nutrients and metabolites between the RPE and photoreceptors (Ishikawa et al., 2015). The IPM is also thought to participate in photoreceptor disk turnover, retinoid transport, calcium buffering, photoreceptor alignment, adhesion of the retina, and cell-to-cell communication, including growth factor presentation (Lazarus and Hageman, 1992; Strauss, 2005; Garlipp et al., 2012; Ishikawa et al., 2015).
The IPM is structurally composed of proteoglycans and glycosaminoglycans (GAG), such as chondroitin sulfate (ChS) and hyaluronic acid (HA; Tien et al., 1992; Hollyfield et al., 1998; Hauck et al., 2005; Clark et al., 2011; Keenan et al., 2012). The IPM does not contain commonly occurring ECM components, such as collagen, or large quantities of ChS proteoglycans, such as aggrecan and neurocan (Ishikawa et al., 2015). Instead, the IPM is composed of two unique and structurally similar ChS 
Table 1. List of names and specifications of antibodies and lectins used in this work

\begin{tabular}{|c|c|c|c|c|c|}
\hline Name & Host & Against & Dilution & Source & Catalog \\
\hline SPARC (G-11) & Mouse & IMPG1/SPARC & $1: 2000$ & Santa Cruz Biotechnology & SC-377366 \\
\hline Anti-IMPG2 & Rabbit & IMPG2/SPARCAN & $1: 1000$ & Sigma-Aldrich & HPA008779 \\
\hline Z0-1 & Rabbit & ZO-1 & $1: 500$ & Thermo Fisher Scientific & $61-7300$ \\
\hline $\mathrm{G} \alpha \mathrm{t} 2(\mathrm{I}-20)$ & Rabbit & Cone transducin & $1: 1000$ & Santa Cruz Biotechnology & SC-390 \\
\hline Iba1 & Rabbit & Iba1 protein & $1: 1000$ & Wako & 019-19741 \\
\hline $3 \mathrm{~B} 3$ & Mouse & Chondroitin 6 sulfate & $1: 100$ & Amsbio & $270433-C S$ \\
\hline GFAP-Cy3 & Rabbit & GFAP & $1: 1000$ & Sigma-Aldrich & $C-9205$ \\
\hline PNA & & $\mathrm{N}$-acetylgalactosamine & $1: 1000$ & Vector Laboratories & B-1075 \\
\hline WGA-680 & & $N$-acetyl-o-glucosamine & $1: 1000$ & Thermo Fisher Scientific & W32465 \\
\hline WGA-488 & & $N$-acetyl-D-glucosamine & $1: 1000$ & Thermo Fisher Scientific & W11261 \\
\hline
\end{tabular}

proteoglycans, the interphotoreceptor matrix proteoglycan 1 (IMPG1) and IMPG2. IMPG1 is also referred to as SPACR (sialoprotein associated with cones and rods) or IPM150 (Kuehn and Hageman, 1999a; Lee et al., 2000; Hollyfield et al., 2001). The other commonly used names for IMPG2 include SPACRCAN (sialoprotein associated with cones and rods proteoglycan) or IPM200 (Kuehn and Hageman, 1999b; Acharya et al., 2000; Hollyfield et al., 2001; Chen et al., 2003). Both proteins are synthesized in the photoreceptors, and their expression levels correlate with the maturation of photoreceptor OS (Foletta et al., 2001; Felemban et al., 2018). IMPG2 is larger than IMPG1 with a putative transmembrane domain that may aid in the attachment of IMPG2 to the cell membrane (Fig. 1A). Both IMPG molecules are modified by ChS and have predicted attachment sites for HA (Hollyfield et al., 1999; Acharya et al., 2000; Chen et al., 2003).

Humans with mutations in IMPG1 or IMPG2 develop vision loss characterized by the accumulation of material in the subretinal space. Subretinal lesions associated with IMPG mutations have been observed in certain eye diseases, such as adult-onset foveomacular vitelliform dystrophy (AFVD), subretinal drusenoid deposits (SDDs), and age-related macular degeneration (AMD; Bandah-Rozenfeld et al., 2010; Zweifel et al., 2011; Boddu et al., 2014; Meunier et al., 2014; van Huet et al., 2014; Chowers et al., 2015; Wilde et al., 2016; Brandl et al., 2017; Guziewicz et al., 2017). Despite the implied role of IMPG1 and IMPG2 proteoglycans for our vision, little is known about the function of these proteins or the pathophysiology of the disease caused by mutations in these IMPGs. In this work, we delved into the IPM physiology by focusing on the role of IMPG1 and IMPG2 as well as into the pathophysiological mechanisms underlying visual impairment caused by defects in these proteins. For this purpose, we developed three global knock-out (KO) mouse lines, IMPG1 $1^{-1-}$, IMPG $2^{-1-}$, and IMPG1-IMPG2 $2^{-1-}$ double KO. Our results indicate that IMPG2 proteoglycan present at the IS is required for the localization of IMPG1 in the OS. In the absence of IMPG2, IMPG1 accumulated between the OS and RPE leading to subretinal lesions, microglial activation, and a decrease in visual function.

\section{Materials and Methods}

\section{Animal model}

The animal models used in this study were generated by Cripsr-Cas9 technology as described by Moye et al. (2018). Briefly, a 20 nt singleguide RNA (sgRNA) was transcribed from a 69 nt sequence containing a T7 promoter, and the desired target sequence against IMPG1 (5'-GCG GCC TCT AAT ACG ACT CAC TAT AGG GGA TCT TTT GGT TCG AAG CTT GTT TTA GAG CTA GAA ATA GCA-3') and IMPG2 (5' GCG GCC TCT AAT ACG ACT CAC TAT AGG GGG CGT GAT GAA TAT CGT CAC GTT TTA GAG CTA GAA ATA GCA-3'). The transcription and cutting efficiency of the sgRNA was assessed using a
Clontech kit (catalog \#631438). In vitro transcribed sgRNA along with Invitrogen Cas9 nuclease (catalog \#B25641, Thermo Fisher Scientific) were injected into pronuclei of B6D2F1 blastocysts. Heterozygote animals were backcrossed with C57BL/6J (The Jackson Laboratory). All animals were generated at the Transgenic Core of West Virginia University and did not contain $r d 1$ or $r d 8$ alleles. IMPG1 and IMPG2 KO animals were crossed to produce IMPG1-IMPG2 double KO. The animals were maintained under $12 \mathrm{~h}$ light/dark cycles with food and water provided ad libitum. Both sexes were used in all experiments. All experimental procedures involving animals in this study were approved by the Institutional Animal Care and Use Committee of West Virginia University.

\section{Immunohistochemistry}

Mice were killed by $\mathrm{CO}_{2}$ exposure, and eyes were enucleated. A small hole on the cornea was made, and the enucleated eyes were placed in $4 \%$ paraformaldehyde (PFA) for $30 \mathrm{~min}$. The cornea and lens were removed after fixation, and the resulting eye cup was further fixed for $30 \mathrm{~min}$. The eyecups were then washed three times for 5 min each in PBS and left in PBS containing $20 \%$ sucrose overnight. The eyecups were placed in a 1:1 mix of $20 \%$ sucrose and optimal cutting temperature compound (Sakura) for $2 \mathrm{~h}$ before being flash frozen in optimal cutting temperature compound and stored at $-80^{\circ} \mathrm{C}$.

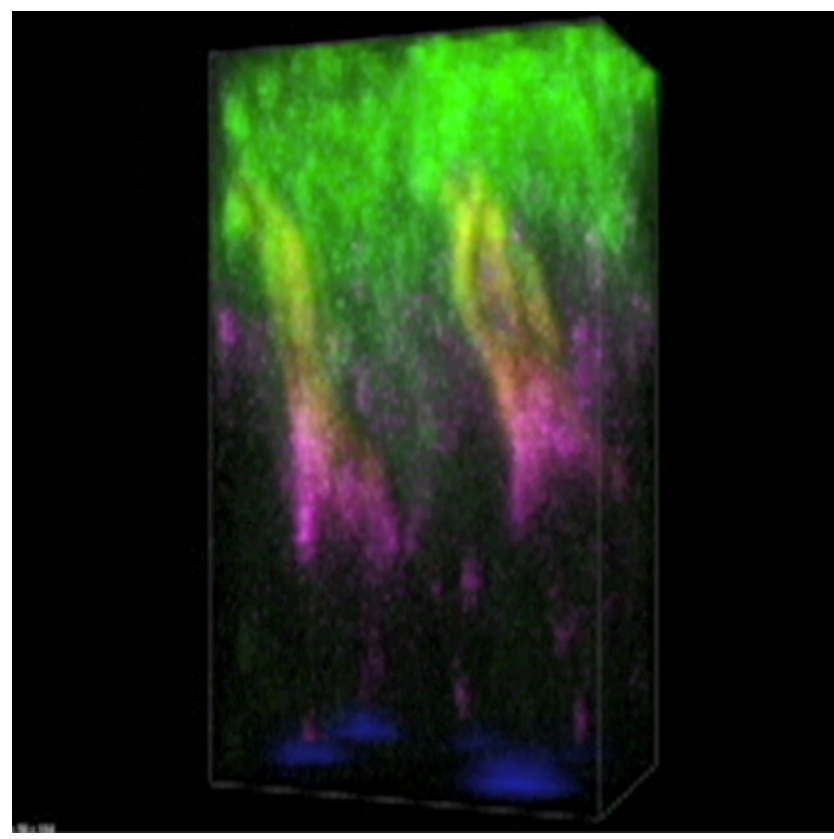

Movie 1. Wild-type retina flat-mount 3D reconstruction centered on cone, and stained with PNA (red), IMPG1 (green), IMPG2 (magenta), and DAPI (blue). PNA colocalizes with IMPG2 around the IS, and with IMPG1 around the OS. IMPG1 stains weakly at the IS in association with IMPG2 proteoglycan. [View online] 
A

TGA

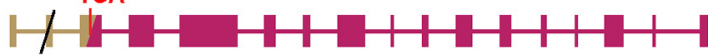

\section{IMPG2}

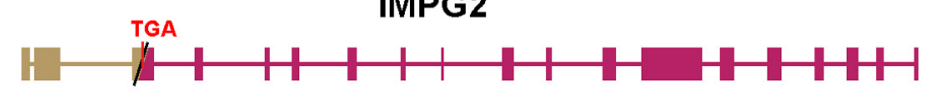

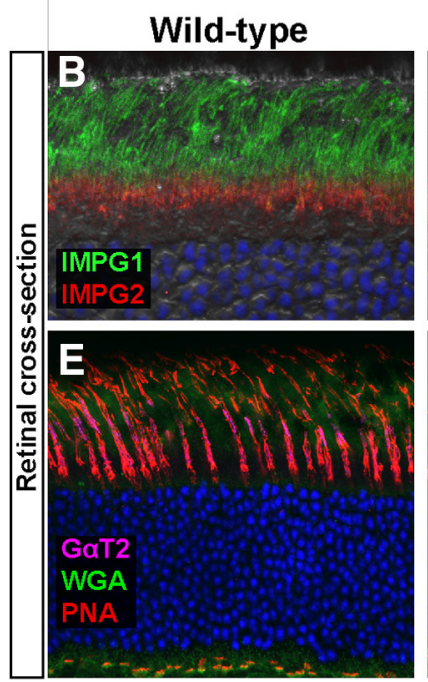
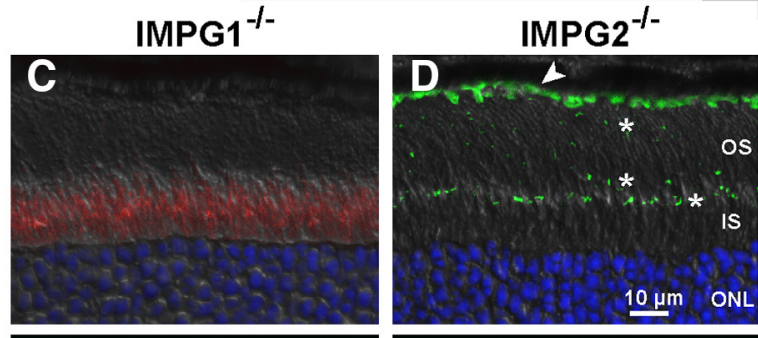

K
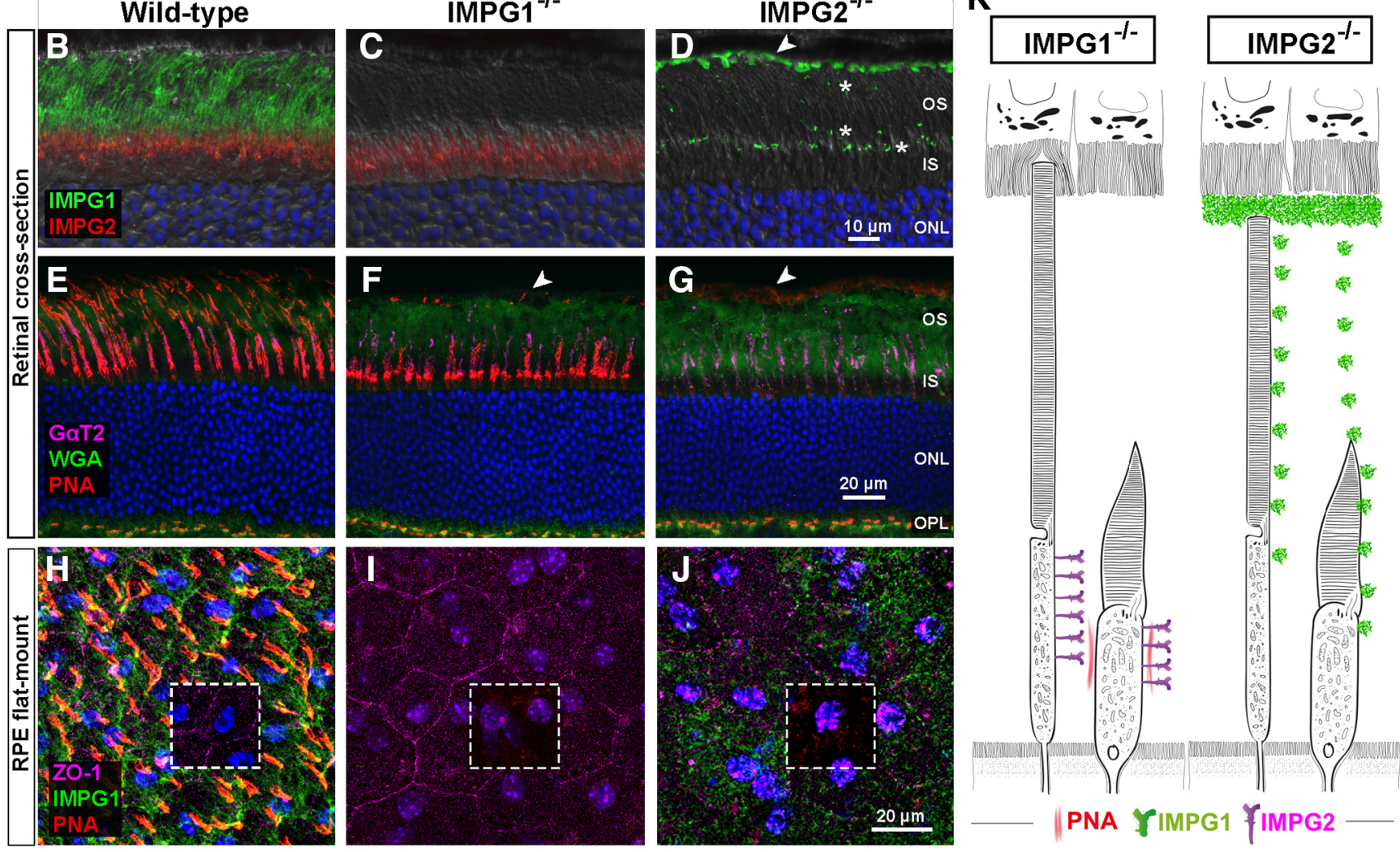

Figure 2. Mislocalization of IMPG1 in the absence of IMPG2. $A$, Schematic representation of the change induced in the IMPG1 and IMPG2 knock-out mice. Induced deletions are marked by the forward slash (/) symbol and predicted premature termination is represented by TGA codon. Pink-colored boxes represent untranslated exons in the mutants beyond the TGA codon. $\boldsymbol{B}-\boldsymbol{J}$, Immunohistochemistry of the proteoglycans IMPG1, IMPG2, and PNA lectin from wild-type (left), IMPG1 (middle), and IMPG2 KO (right) mice. C, IMPG1 KO mice show the absence of IMPG1 (green) and preserved IMPG2 (red) staining at the IS. D, Lack of IMPG2 staining in IMPG2 KO mice and accumulation of IMPG1 scattered throughout the OS (asterisks) and at the boundary between the RPE and the OS (arrow). $\boldsymbol{F}$, Loss of cone-specific lectin PNA (red) at the OS of IMPG1 KO mice. Note a faint PNA staining at the RPE-0S boundary (arrow). In contrast, the lectin WGA (green) and the cone-cell marker, cone transducin protein G $\alpha$ t2 (magenta), staining are unaltered. G, In IMPG2 KO retinas, WGA and Gat2 staining extend normally, but the staining of PNA is absent or faint, while aberrant PNA staining was found at the boundary between RPE and OS (arrow). PNA staining in unaltered at the outer plexiform layer (OPL) in all groups. $\boldsymbol{H}-\boldsymbol{J}$, RPE flat-mount stained against PNA (red), IMPG1(green), and Z0-1 (magenta). $\boldsymbol{H}$, Wild-type mice have a structured IMPG1 and PNA above RPE cells. I, IMPG1 KO lacks IMPG1 and shows a faint PNA stain above RPE. J, IMPG2 KO shows a dense, unorganized IMPG1 staining with faint PNA above the RPE cells. The boxes in dotted lines delineate an area eliminating the signals from IMPG1 and PNA in $\boldsymbol{G}$, and in $\boldsymbol{H}$ and $\boldsymbol{I}$ after removing the signals from IMPG1 and $\mathbf{G} \alpha \mathrm{T}$. Pictures are representative of three independent experiments. All images were obtained at $60 \times$ magnification using 45-d-old mice. $\boldsymbol{K}$, Schematic representation of the findings obtained from IMPG1 and IMPG2 K0 mice.

The frozen retinal tissue was sectioned with $16 \mu \mathrm{m}$ thickness using the Leica CM1850 cryostat, mounted on Superfrost Plus Slides (Thermo Fisher Scientific) and stored at $-20^{\circ} \mathrm{C}$. Retinal sections were washed in PBS and incubated for $1 \mathrm{~h}$ in blocking buffer (PBS with 5\% goat sera, $0.5 \%$ Triton X-100, $0.05 \%$ sodium azide) at room temperature and then treated with primary antibody overnight at $4^{\circ} \mathrm{C}$. On the next day, the sections were washed three times for $10 \mathrm{~min}$ in PBS-T (0.5\% Triton X-100). This was followed by the addition of secondary antibody for $2 \mathrm{~h}$ at room temperature. The dilutions used for lectins, primary antibodies, and secondary antibodies are presented in Table 1 . Sections were washed three times per $10 \mathrm{~min}$ in PBS-T and then sealed with a coverslip after the addition of Life Technologies ProLong Gold (Thermo Fisher Scientific).

RPE and retina flat-mount preparations were made from enucleated eyes, as described above. Briefly, the cornea was removed after $30 \mathrm{~min}$ fixation in $4 \%$ PFA, and the resulting eyecups were fixed for 2 more hours. After fixation, the eyecup was cut from the periphery toward the optic nerve at four places. The retina and RPE were carefully detached under a dissection microscope. The tissue was further processed as described above.

Images were acquired and processed with a Nikon C2 laser-scanning confocal microscope using excitation wavelengths of 405, 488, 543, and $647 \mathrm{~nm}$. Four sections were imaged for each sample, and data were derived from three independent experiments using littermates as controls.

For analyzing retinal morphology by hematoxylin and eosin (H\&E) staining, enucleated eyes were shipped in fixative for serial cross sectioning and H\&E staining to Excalibur Pathology. Images were taken with an Olympus VS120 slide scanner.

\section{Photoreceptor ultrastructure}

Mice from three different littermates per group were killed by $\mathrm{CO}_{2}$, and eyes were carefully enucleated. Eyes were first fixed in $2 \%$ paraformaldehyde, $2.5 \%$ glutaraldehyde, and $0.1 \mathrm{~m}$ cacodylate buffer, at $\mathrm{pH} 7.5$ for $30 \mathrm{~min}$. The cornea and lens were removed, and the fixation of the tissue was continued in the same buffer at room temperature for $48 \mathrm{~h}$ under rotation. Following dissection, toluidine blue staining, embedding, and transmission electron microscopy scanning were performed at the Robert P. Apkarian Integrated Electron Microscopy Core at Emory University (Atlanta, GA), as described previously (Dilan et al., 2019).

Spectral domain optical coherence tomography

Animals were anesthetized by isoflurane $(2.0 \%$ isoflurane at $1 \mathrm{~L} / \mathrm{min}$ oxygen flow rate), and eyes were topically dilated with a 1:1 mixture of 


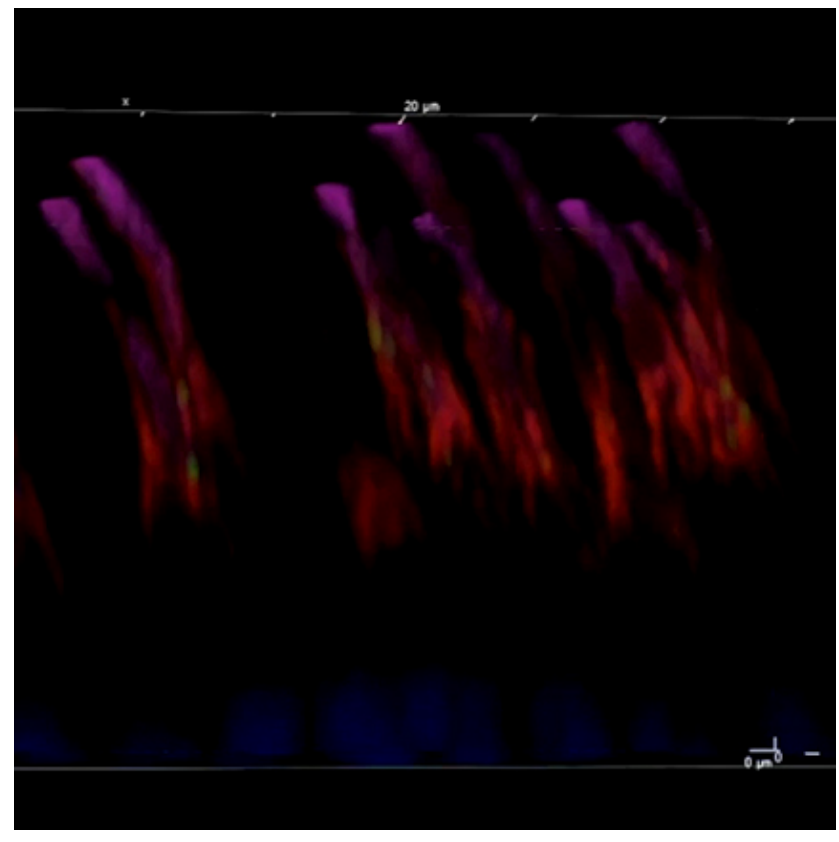

Movie 2. 3D reconstruction of the flat-mount retina from wild-type, IMPG1, and IMPG2 KO mice. Stained with PNA (red), IMPG1 (green), Gat2 (magenta), and DAPI (blue). Wildtype retina shows $G \alpha t 2$ in the center of the cone photoreceptors surrounded by PNA and IMPG1. In IMPG1 K0 retina, PNA localizes just at the base of the cone, and G $\alpha \mathrm{t} 2$ antibody stains the cone OS. In IMPG2 KO retina, G $\alpha$ t2 stains the cone OS surrounded by scattered staining pattern for IMPG1. [View online]

tropicamide:phenylephrine hydrochloride. The body temperature of the animals was maintained by placing them on a heating pad. Ophthalmic gel (Genteal, Alcon) was applied to the cornea to avoid drying. Using Envisu R2200 high-resolution optical coherence tomography (OCT) equipment, we captured images covering a surface area of $1.4 \times 1.4 \mathrm{~mm}$ centered at the optic nerve with $100 \mathrm{~B}$-scans per eye with 1000 points of resolution per B-scan.

\section{Electroretinogram}

After $24 \mathrm{~h}$ of dark adaptation, adult mice were anesthetized in an induction chamber with isoflurane (1.5\% at $1 \mathrm{~L} /$ min oxygen flux). After induction, pupils were dilated with a 1:1 mixture of tropicamide:phenylephrine hydrochloride for $10 \mathrm{~min}$ before recording. The mice were placed on a heated platform and connected to an anesthesia cone mask at the same oxygen flux. The reference electrode was placed on the neck, and the ground electrode on the left leg. Full scotopic flash intensity electroretinogram (ERG) was performed with white light in dark-adapted mice. For the photopic ERG mode, a white background light of $30 \mathrm{~cd} / \mathrm{m}^{2}$ for $10 \mathrm{~min}$ was used to adapt animals to light, and increasing flash intensities were used to stimulate the eyes. ERGs were measured using a Celeris system from Diagnosys with fully integrated electrodes built into the stimulator.

Experimental design and statistical analysis

All analysis was performed on age-matched mutants and littermate wild-type (WT) control animals. We used animals of both sexes and did not find any sex-dependent changes in the phenotypes. ERG responses were analyzed with two-way ANOVA and then Tukey's post hoc test for comparison of means between groups. The activated microglial count (Iba1) was analyzed with Nikon NIS-Elements software with one-way ANOVA and then Tukey's post hoc test for comparison of means between groups. All data are presented as the mean \pm SEM.

\section{Results}

Localization of photoreceptor-specific IMPG proteoglycans IMPG1 and IMPG2 are photoreceptor-specific proteins within the retina (Kuehn and Hageman, 1999a; Acharya et al., 2000). A closer inspection of the IMPG protein sequence shows the presence of an $\mathrm{N}$-terminal signal sequence, suggesting that these proteins are exported from the photoreceptor cells (Fig. 1A). To determine the location of IMPGs in the murine retina, we performed an immunohistochemistry (IHC) analysis (Fig. 1). We found distinct patterns of IMPG1 and IMPG2 staining surrounding the photoreceptor cells. Specifically, we find IMPG2 in the distal regions (closer to the OS) of the IS (Fig. 1B) and IMPG1 in the area surrounding the distal IS and the OS (Fig. 1C). A magnified image shows IMPG1 and IMPG2 surrounding both rods and cones (Fig. 1E-G). The lectin peanut agglutinin (PNA), an established cone-specific IPM marker, colocalizes with both IMPG1 and IMPG2 (Fig. 1E,F, arrows).

To highlight the differences in localization of IMPGs between rods and cones, we performed flat-mount transverse sections of the retina and $3 \mathrm{D}$ reconstruction focusing on single cones (Fig. $1 H-J$, and corresponding subpanels). The IMPG2 stain appears at $10 \mu \mathrm{m}$ distal from the outer nuclear layer (ONL) as rings surrounding the cones $(\mathrm{H} 3)$. At $13 \mu \mathrm{m}$, IMPG2 appears as smaller rings around rods in between cones (H2). Further up at $18 \mu \mathrm{m}$, IMPG2 staining is diffuse (H1). In contrast, faint IMPG1-stained, ring-like structures can be found around cones at $10 \mu \mathrm{m}$ above the ONL (I3) where it colocalizes with IMPG2 (J3). At $13 \mu \mathrm{m}$, robust staining for IMPG1 around cones and faint IMPG1 staining around rods was noted. Further up at the OS level, the IMPG1 staining is consistently strong. The superimposition of IMPG1 and IMPG2 images (Fig. 1J) highlight the transition from IMPG2 to IMPG1 and the different planes between cone and rod IMPGs. In this sense, Figure 1J.2 shows the cone-IMPG1 in green passing through a magenta honeycomb shape of rodIMPG2. A 3D video reconstruction shows IMPG localization in wild-type retinas to visualize the spatial relationships among IMPG proteoglycans and between rods and cones (Movie 1). In summary, our results demonstrate that IMPG1 and IMPG2 have distinct pattern distributions in the IPM, and both cones and rods are surrounded by IMPG proteoglycans.

\section{IMPG1 location depends on IMPG2}

To study the role of IMPG1 and IMPG2 in retinal physiology, we generated KO mouse models using CRISPR-Cas9 technology. In the IMPG1 mutant, Cas9 nuclease excision led to a $10 \mathrm{nt}$ deletion in exon 2, producing a premature stop codon in exon 3 . In the IMPG2 mutant, a $7 \mathrm{nt}$ deletion in exon 3 led to a premature termination on the same exon. In both cases, we expected nonsense-mediated decay and consequent loss of IMPG1 and IMPG2 proteins (Fig. $2 A$ ). The IHC analysis confirmed the absence of IMPG1 or IMPG2 proteoglycans in IMPG1 and IMPG2 $\mathrm{KO}$ mice, respectively. These studies demonstrate the specificity of the antibodies used and validate the knock-out models (Fig. $2 B-D)$.

The distribution of IMPG2 at the IS was unaltered in the absence of IMPG1 (Fig. 2C). Interestingly, in IMPG2 KO mice, IMPG1 accumulates at the boundary between the OS and RPE, in addition to a scattered staining pattern through the OS (Fig. 2D). Furthermore, the cone-specific marker PNA disappeared around the OS in the absence of IMPG1 but remained at the IS region mimicking the IMPG2 staining pattern (Fig. $2 C, F$ ). In IMPG2 KO retinas, PNA was mislocalized and located at the RPE-OS boundary, like IMPG1 (Fig. 2D,G). A 3D video reconstruction of flat-mounted retina from wild-type and mutant mice illustrates the absence of PNA in the OS of IMPG1 KO and the sporadic presence of IMPG1 in IMPG2 KO mice (Movie 2). 

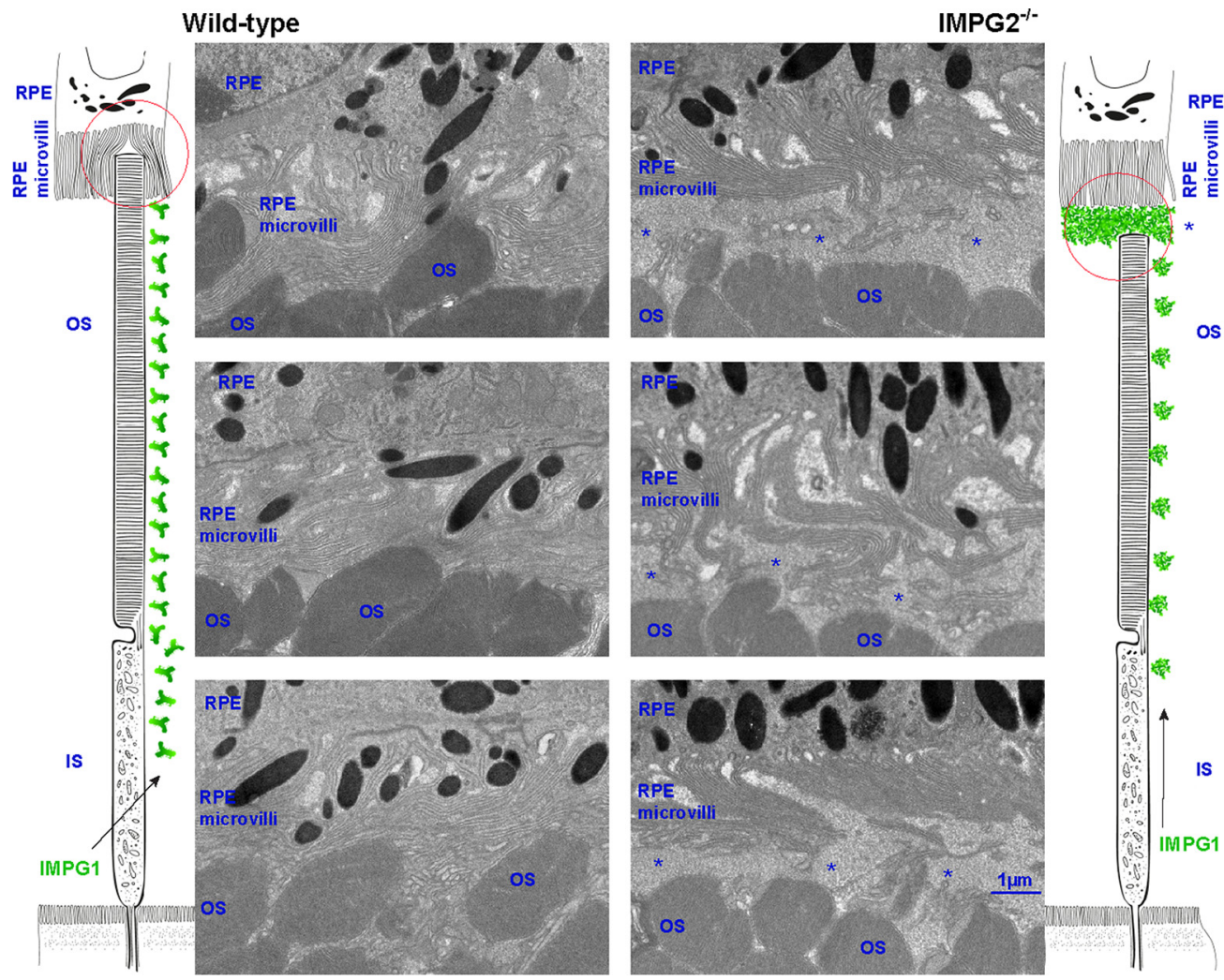

Figure 3. Anomalous material accumulation between photoreceptor OS and RPE in the absence of IMPG2. Electron microscopic images of the retina from wild-type mice exhibit a close contact between $0 S$ and microvilli of the RPE. In contrast, the retinas from IMPG2 K0 animals show a separation between $0 S$ and RPE filled by an unstructured material (asterisks). Retinal tissue used was collected from 5 -month-old mice $(n=3)$.

To further study the mislocalized IMPG1 and PNA at the boundary between the OS and the RPE, we performed an IHC analysis on flat-mount RPE cells. Wild-type tissue shows an organized and regularly spaced distribution of IMPG1 and PNA in contact with the RPE cells (Fig. 2H). However, flat-mount from IMPG1 KO mice shows the absence of IMPG1 and a diffuse pattern of PNA staining (Fig. 2I). IMPG2 KO mice reveal a dense unstructured IMPG1 and PNA staining on the RPE surface (Fig. 2J). These results show a crucial role for IMPG2 proteoglycan in the distribution of IMPG1 across the retina and the direct relationship between IMPG proteoglycans and PNA lectin staining.

\section{Subretinal material accumulation in IMPG2 KO mice}

We speculated that the accumulation of IMPG1 proteoglycan in IMPG2 KO retina affects the interaction between RPE microvilli and the photoreceptor OS. A magnified view by electron microscopy shows the accumulation of heterogeneous material that separates the RPE microvilli from the OS in IMPG2 KO mice (Fig. 3). In contrast, wild-type animals show the RPE microvilli in intimate contact with the OS. The IMPG1 KO retinas did not show any detectable changes in the RPE-OS interface.

\section{IMPG2 KO mice develop subretinal lesions}

Patients with mutations linked to IMPG proteoglycans develop visual impairment with punctate subretinal macular bodies detected by spectral domain OCT (SD-OCT). Therefore, we investigated the retinal morphology using SD-OCT imaging in IMPG2 KO mice at 5 months. We found that most animals develop one or two hyper-reflective clumps in the subretinal space (Fig. 4). The clumps resemble the subretinal vitelliform lesions found in humans (Fig. 4A,A.1,A.3, arrows; Meunier et al., 2014). The histologic analysis of the retina examined by SD-OCT confirmed these findings and showed a prominent bulge of fibrous materials separating the retina from the RPE and cells infiltrating in the subretinal space (Fig. $4 B-D$ ). Moreover, the analyses of serial sections identified multiple, smaller subretinal material accumulations in IMPG2 $\mathrm{KO}$ retinas that were difficult to detect using the in vivo SD-OCT technique (Fig. 4A.2,C). In contrast to IMPG2 KO retinas, no detectable alterations or subretinal accumulation were observed by OCT or histologic analyses in littermate wild-type controls or IMPG1 KO animals. These results show that IMPG2 KO mice develop lesions that resemble the subretinal lesions found in humans with IMPG mutations.

\section{Subretinal lesions are rich in IMPG1 proteoglycan, activated microglia, and reactive macroglia}

Next, we hypothesized that the subretinal lesions are due to excessive accumulation of IMPG1 proteoglycan. To study the composition of these lesions, we stained cross sections of subretinal lesions with IMPG1 antibody. The lesions showed distinct staining for IMPG1 in between the RPE and the OS (Fig. 4E,E.I,F.1, G.1). Additionally, the lesions show cell nuclei stained with DAPI (Fig. 4E.2,E.3, rectangles). Subsequently, we explored whether the cell nuclei in the subretinal lesions could be due to retinal microglia migrating to the area. Microglia were detected using an antibody against a microglia/macrophage-specific, calcium-binding protein (Iba1). In healthy retinas, microglial cells $\left(\mathrm{Ibal}^{+}\right)$displayed a ramified morphology and are located specifically in the ganglion cell layer, as well as the inner and outer plexiform layers, as previously reported (Okunuki et al., 2018). Interestingly, in IMPG2 KO mice we observed $\mathrm{Ibal}^{+}$cells 


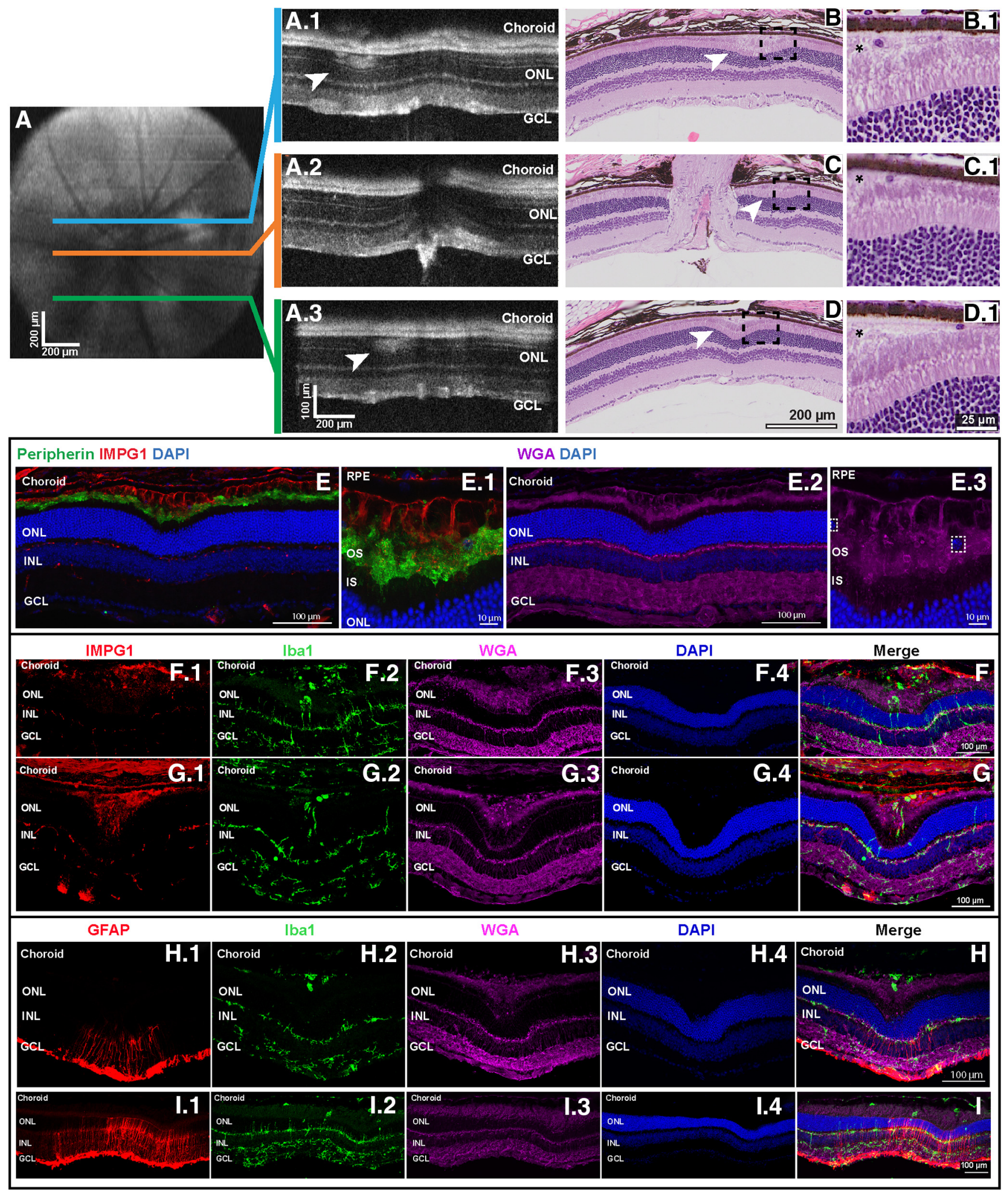

Figure 4. IMPG2 KO mice develop subretinal lesions. A, Representative study of in vivo SD-OCT in IMPG2 KO. A.1-A.3, En face image centered at the photoreceptors layer with color-indicated orientations of cross-sectional B-scans. A.1, A.3, Subretinal hyper-reflective nodules compromising the IS and OS layers, clinically referred to as subretinal vitelliform lesions in humans (arrowheads). $\boldsymbol{B}-\boldsymbol{D}$, H\&E serial cross sections of the same SD-OCT in $\boldsymbol{A}$. The subretinal lesions (arrowheads) exhibit a dome-shaped deformation on the retina. Magnification of the lesions shows an accumulation of eosinophil material between the $0 \mathrm{~S}$ and the RPE, with the presence of infiltrated cells (asterisks in B.1, C.1, and D.1). Data are representative of 5-month-old IMPG2 KO mice $(N=11)$ with subretinal lesions. $\boldsymbol{E}$, Birds-eye view of a subretinal lesion stained against IMPG1 (red) and the OS marker peripherin (green), and a magnified view of the subretinal lesion (E.1). E.2, E.3, Same retina stained with WGA lectin (magenta) and DAPI showing the nuclei of the infiltrated cells (white box). $F, \mathbf{G}(\mathbf{1}-4)$, Microglia stained with Iba1 (green) antibody have migrated into the $0 N L$, IS, OS, and the subretinal space. $\boldsymbol{H}, \boldsymbol{I}(\mathbf{1 - 4})$, GFAP reactivity (red) restricted to the subretinal lesion. 

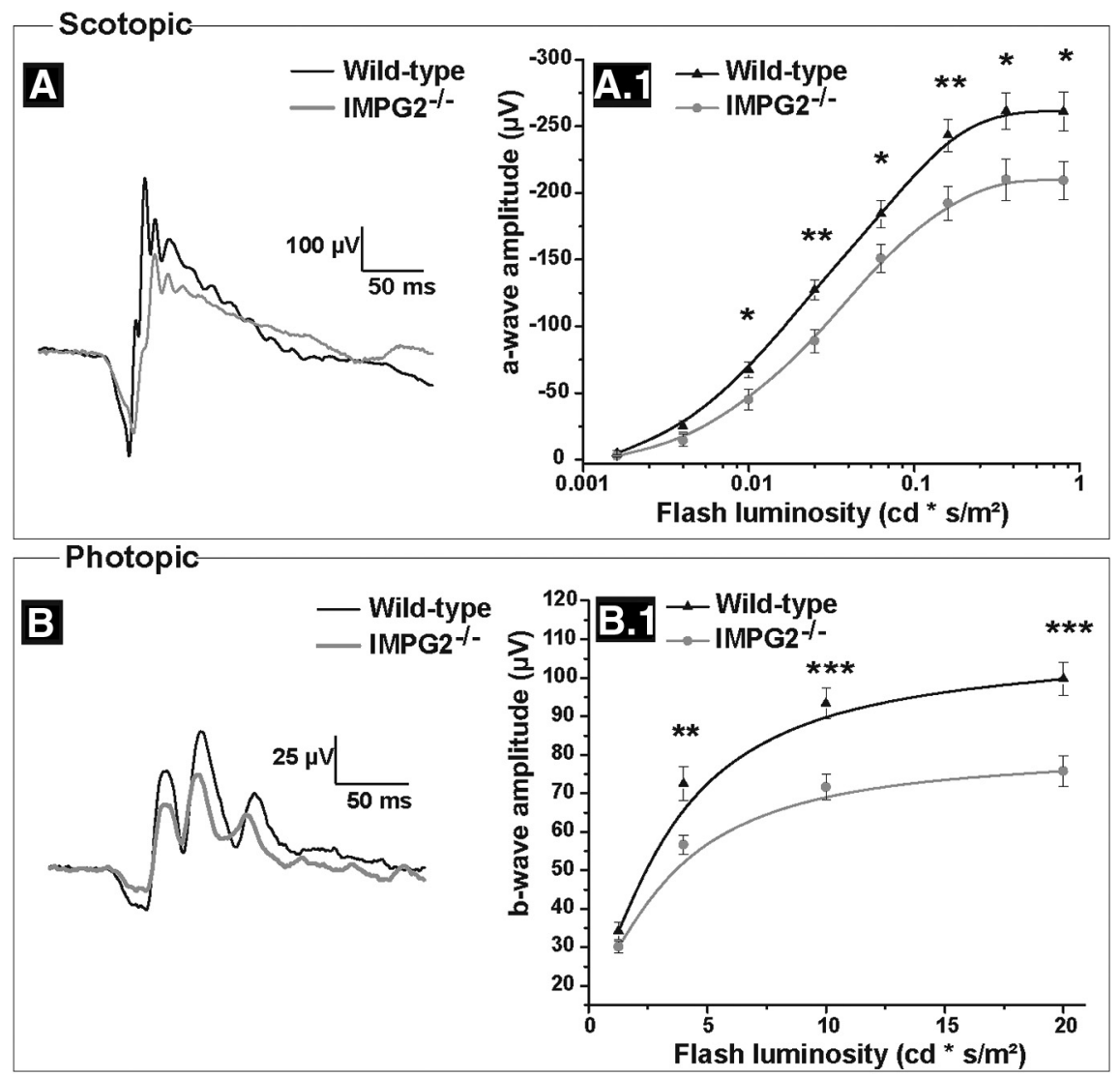

Figure 5. Reduced ERG response in 8-month-old IMPG2 KO mice. $\boldsymbol{A}, \boldsymbol{B}$ Scotopic (rods) at $0.16 \mathrm{~cd} / \mathrm{s} / \mathrm{m}^{2}$ flash intensity and photopic (cones) at $10 \mathrm{~cd} / \mathrm{s} / \mathrm{m}^{2}$ flash intensity ERG responses in wild-type (black line) and IMPG2 knock-out (gray line) mice. A.1, Scotopic A-wave sensitivity curve. B.1, Photopic B-wave responses from light-adapted mice to increasing light intensities. Data points are the mean \pm SEM of $N=16$ eyes, two-way ANOVA (8 mice/group from three different littermates). ${ }^{*} p<0.05 ;{ }^{* *} p<0.01 ;{ }^{* * *} p<0.001$.

invading the photoreceptor layers and the subretinal lesions (Fig. $4 F, G, H .2, I .2)$. Then, we investigated whether Müller glia also react to the subretinal lesion by staining against the glial fibrillary acidic protein (GFAP). Müller reactive gliosis was exclusively found in the retina proximate to the lesions (Fig. 4H,I).

Combined, these results indicate that the accumulation of IMPG1 proteoglycan is a key component in the formation of the subretinal lesions. Furthermore, the lesion leads to reactive gliosis and microglia migration to the photoreceptor layer and subretinal space.

Photoreceptor function is reduced in the absence of IMPG2 To study the impact of the material accumulated between the OS and RPE on the photoreceptor function in the animals that lack IMPG2, we used ERGs (Fig. 5). The ERG technique measures changes in the electric field of the eye in response to light stimulation and is capable of distinguishing rod or cone photoreceptor responses by preadapting the animals to dark (scotopic conditions) or light (photopic condition), respectively. IMPG2 KO mice show a significant decrease in scotopic and photopic ERG responses at different light intensities at 8 months (Fig. 5). In contrast, IMPG1 $\mathrm{KO}$ animals did not show any changes in the ERG responses. In 8month-old animals, the ERG response from IMPG1 KO animals was identical to that from the wild-type littermate controls.

\section{Mice lacking IMPG1 and IMPG2 do not develop subretinal lesions or visual deficits}

To evaluate the role of IMPG1 in the pathology of IMPG2-null animals and to study possible functional redundancy among
IMPGs, we generated IMPG1 and IMPG2 double KO mice. The absence of IMPG1 and 2 proteins in the retina of double $\mathrm{KO}$ mice were confirmed by IHC analysis (Fig. 6A,B). We also stained retinas obtained from double $\mathrm{KO}$ and wild-type mice with the lectin wheat germ agglutinin (WGA) that stains the IPM. Interestingly, IMPG1-IMPG2 double KO retinas did not show material accumulation, subretinal lesions, or changes in the IPM (Fig. 6B). We then focused on cones using the intracellular OS cone marker, cone transducin $\alpha$-subunit G $\alpha$ T2 (green), and the cone-specific extracellular matrix marker PNA lectin (Fig. 6C,D, red). There were no changes in the number of cones in the retina lacking IMPGs as judged by cone transducin expression. However, the PNA staining was absent in the IPM of the IMPG1-IMPG2 double KO (Fig. 6D).

We next used photopic and scotopic ERG to analyze the function of the IMPG1-IMPG2 double KO photoreceptors (Fig. 6E) and in vivo SD-OCT imaging to assess the presence of subretinal lesions (Fig. 6F). Interestingly, these mice did not show any functional deficits or associated subretinal lesions up to 8 months.

Next, we studied the microglial activation of wild-type, IMPG1 KO, IMPG2 KO, and double KO in retinal sections by counting the $\mathrm{Iba}^{+}$cells that migrated to the photoreceptor layers (Fig. 6G). As described earlier, IMPG2 KO retinas showed a significant increase of $\mathrm{Iba}^{+}$cells. In the double knockouts, while there was a reduction in $\mathrm{Ibal}^{+}$ staining, microglial activation was not eliminated (Fig. $6 G)$. 
Wild-type
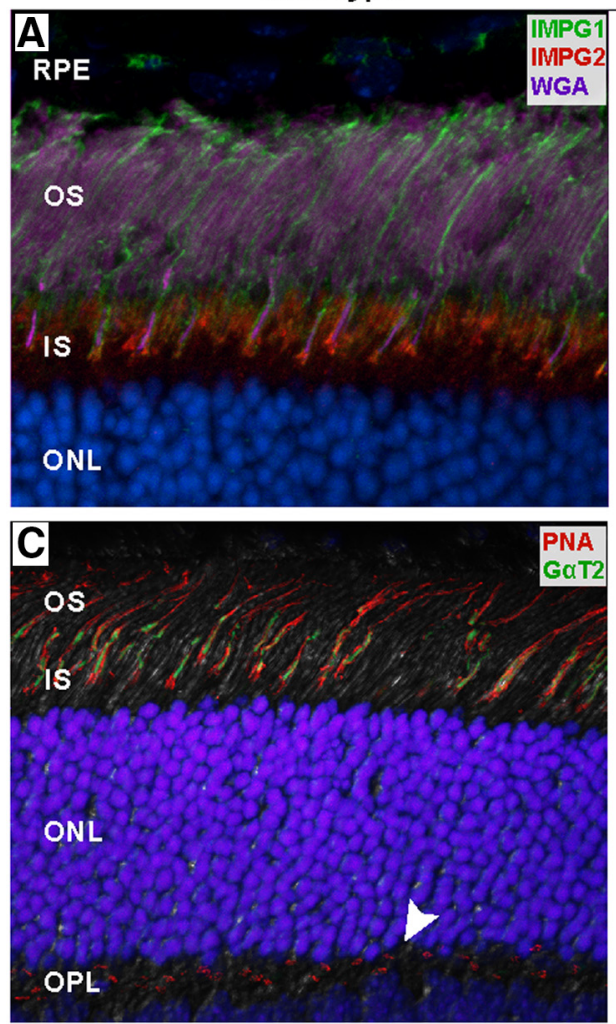

IMPG1-2
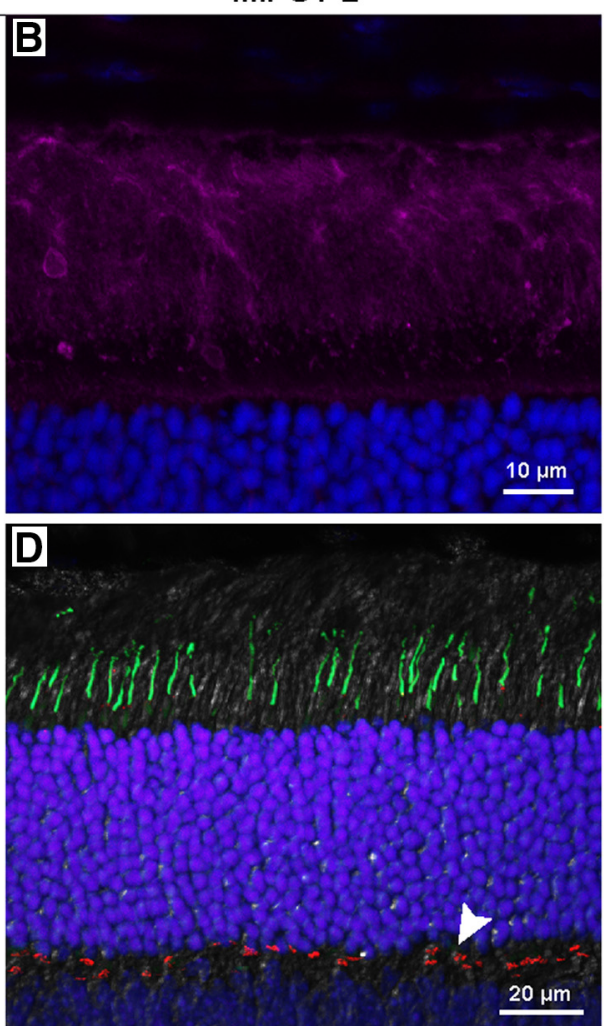

ERG
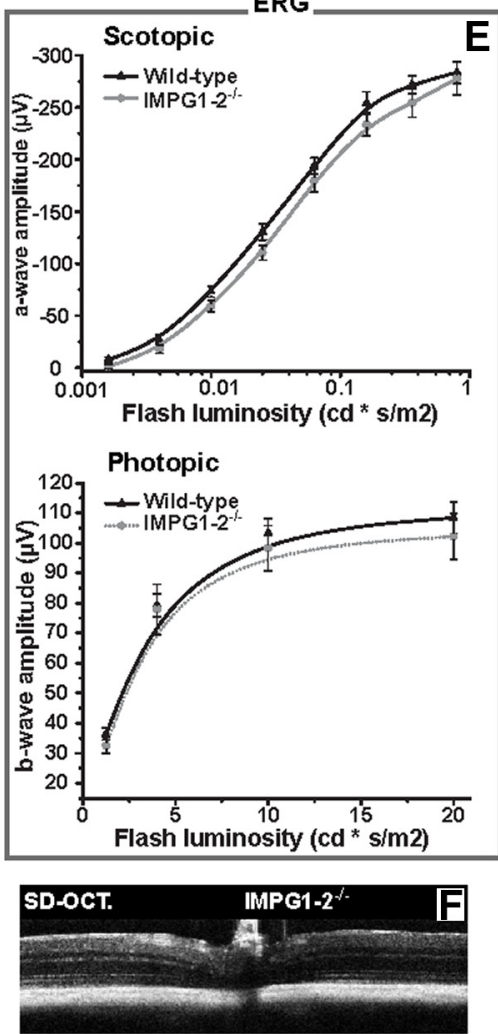
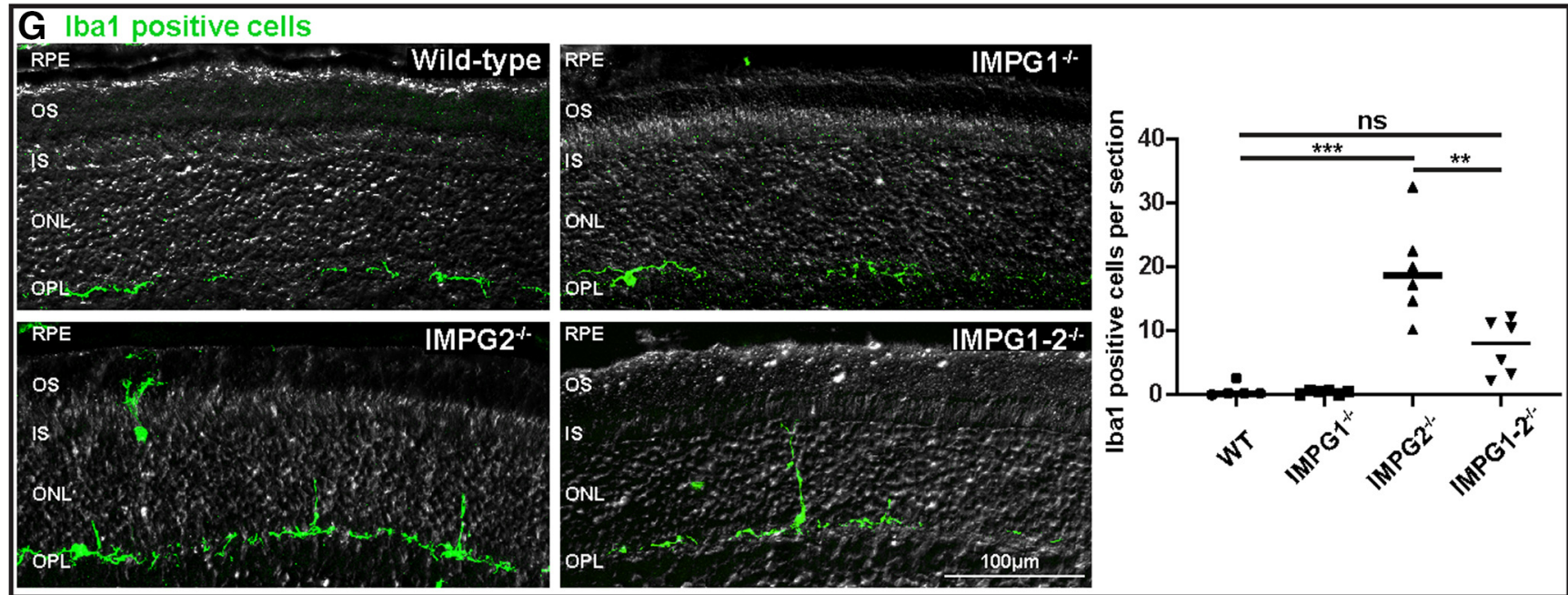

Figure 6. IMPG1-IMPG2 double KO mice show intact retinal structure and function without subretinal lesions. $\boldsymbol{A}, \boldsymbol{B}$, Wild-type $(\boldsymbol{A})$ and IMPG1-IMPG2 double knock-out $(\boldsymbol{B})$ retinas stained against IMPG1 (green), IMPG2 (red), and WGA (magenta). C, Wild-type retina stained with PNA lectin (red) and the cone 0S marker cone transducin G $\alpha \mathrm{T2}$ (green). $\boldsymbol{D}$, Loss of PNA staining surrounding the cones in the retina lacking IMPG1-IMPG2. Interestingly, the staining of the outer plexiform layer (OPL; arrow) is unaltered. Staining for cone transducin (G $\alpha \mathrm{T} 2$ ) shows a normal number of cones in IMPG1-IMPG2 double knockouts. Representative images from three eye sections from three different litters at P45. $\boldsymbol{E}$, Electroretinogram analysis of 8-month-old animals shows no significant differences in scotopic or photopic responses between IMPG1/IMPG2 double mutant and wild-type mice. $\boldsymbol{F}$, SD-0CT representative image of 8-month-old IMPG1/IMPG2 double KO mouse. G, Evaluation of microglial migration to the photoreceptor layers in wild-type, IMPG1, IMPG2, and double KO mice retinas visualized by Iba1 antibody (green) and differential interference contrast (DIC) technique. Quantification of $\mathrm{Iba}^{+}{ }^{+}$cells per retinal sections obtained from a minimum of five different animals from three different litters at 5 months old. Bars are the mean \pm SEM. ns, $p=0.094 ; * * 0.0011 ; * * * 0.0001$.

These results indicate that the cone-specific lectin PNA stains IMPG1 and IMPG2 proteoglycans. In addition, our findings show that the absence of both IMPG proteoglycans does not affect the retinal structure and function. More importantly, removing IMPG1 protein along with IMPG2 reduces the pathologic signs observed in IMPG2 KO, suggesting that the mechanism behind visual impairment in IMPG2 KO mice involves the mislocalization and accumulation of IMPG1.
IMPG1 and IMPG2 are chondroitin sulfate proteoglycans IMPG proteoglycans bind ChS (Lazarus and Hageman, 1992; Hollyfield et al., 1999; Clark et al., 2011). To confirm those results and to obtain an independent assessment of IMPG mislocalization, we investigated the pattern of ChS localization in the IPM of IMPG mutant animals (Fig. 7). ChS consists of hundreds of repeating disaccharide units including chondroitin 6sulfated disaccharide (C6S). To expose the C6S epitopes, we 

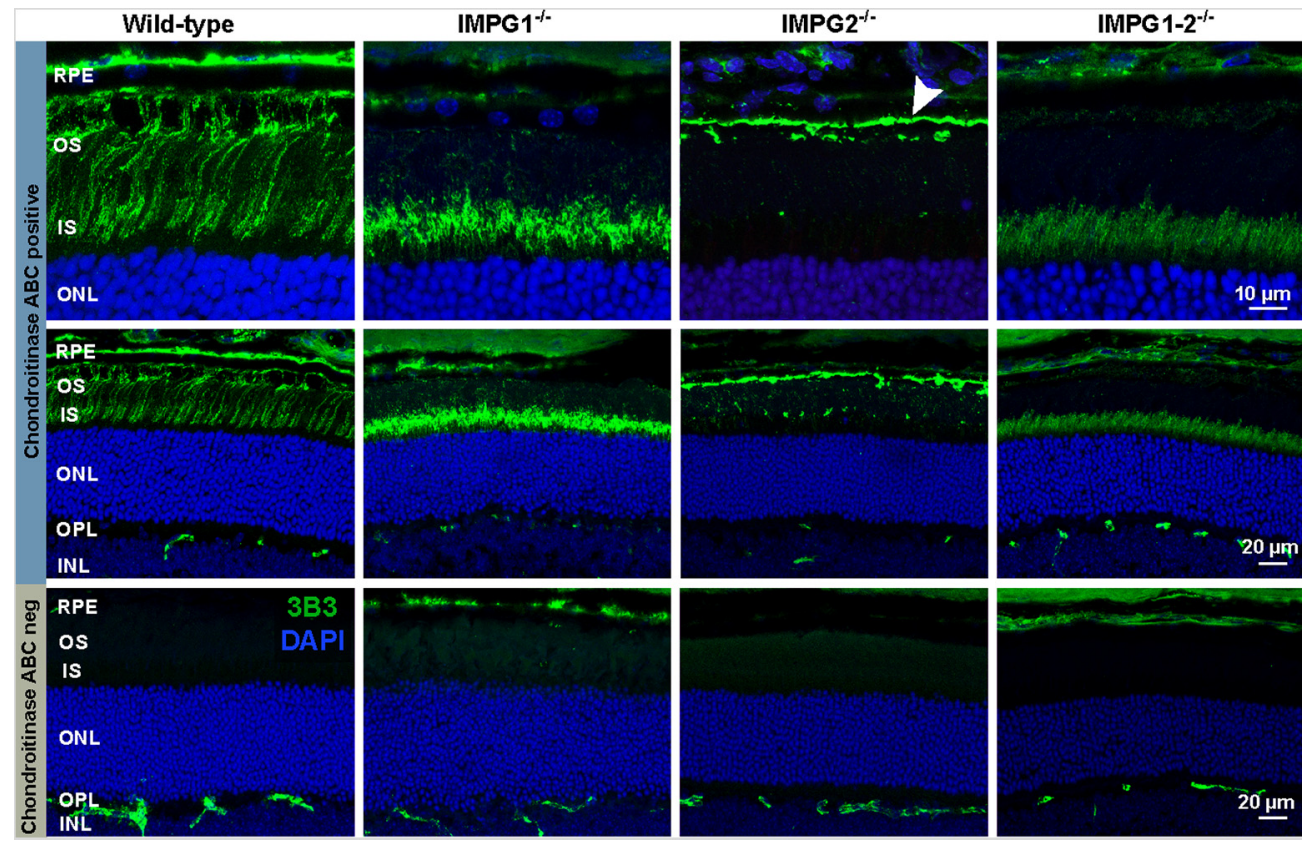

Figure 7. Mislocalization of chondroitin sulfate in the IPM of IMPG1, IMPG2, and IMPG1-IMPG2 double K0 mice. Immunohistochemistry on retinal cross sections from wild-type and IMPGs KO mice stained with the anti-chondroitin sulfate antibody 3B3 (green). Sections treated with chondroitinase ABC (top and middle) or untreated (bottom). Top, 100× magnification; middle, $40 \times$ magnification. Wild-type retina showing 3B3 staining at the outer section of the IS and through the entire OS. In IMPG1 K0 mouse, 3B3 staining is restricted to the IS, while IMPG2 K0 mouse shows ChS accumulation at the OS-RPE boundary (arrowhead) plus a scattered staining pattern along the IPM. Both single mutants mimic the mislocalization staining pattern seen for PNA and IMPG1 in Figure 3. IMPG1/IMPG2 double K0 expresses faint staining for 3B3 at the IS. Bottom, Retinas untreated with chondroitinase ABC do not show $3 B 3$ staining at the IPM. Images processed from three different animals from three different litters at P45.

treated the retinal tissue with chondroitinase $\mathrm{ABC}$ (Couchman et al., 1984; Caterson et al., 1985; Caterson, 2012). Then, we used the monoclonal antibody $3 \mathrm{~B} 3$ that recognizes $\mathrm{C} 6 \mathrm{~S}$ epitopes. The antibody can also stain "native" C6S epitopes in a terminal end of glucuronic acid without chondroitinase $\mathrm{ABC}$ treatment. Retinas without chondroitinase $A B C$ treatment show "native" $3 \mathrm{~B} 3$ epitopes at the outer plexiform layer and sclera but do not show any staining at the IPM (Fig. 7, bottom).

Wild-type retina predigested and stained with $3 \mathrm{~B} 3$ antibody revealed that the IPM of the mouse retinal tissue is rich in $\mathrm{ChS}$, which is localized at the IS and OS of the photoreceptors (Fig. 7, top panels). In the retina lacking IMPG1, ChS is reduced at the OS but shows a robust presence in the IS. On the other hand, in the absence of IMPG2, ChS is mislocalized at the boundary between OS and RPE. In addition, we observed sporadic staining along the OS and at the IS-OS intersection. The observed ChS mislocalization patterns in IMPG mutants are analogous to the PNA and IMPG distributions seen in Figure 2. These results suggest that IMPG proteoglycans are covalently modified by $\mathrm{ChS}$ and that both IMPGs are responsible for the main staining pattern observed for $\mathrm{ChS}$ in the IPM. In addition, the ChS distribution in IMPG2 KO mice points out that the material that accumulates between the OS and RPE is rich in ChS.

Interestingly, in the retina lacking IMPG1 and IMPG2, the ChS staining does not entirely disappear; we instead found faint staining for $\mathrm{ChS}$ at the IS. This finding suggests that other extracellular proteoglycans may compensate for the deficit of $\mathrm{ChS}$ in the absence of both IMPG proteins.

\section{Discussion}

In this study, we used knock-out technology in mice to determine the contributions of IMPG1 and IMPG2 to the structure and function of the IPM. Our data indicate that both IMPG1 and IMPG2 proteoglycans surround the photoreceptors wherein IMPG2 facilitates the localization of IMPG1 in the IPM. Moreover, in the absence of IMPG2, IMPG1 mislocalizes, generating subretinal lesions comparable to those found in humans and likely causing defects in photoreceptor function.

The proteoglycans IMPG1 and IMPG2 are synthesized by photoreceptors (Kuehn and Hageman, 1999a; Lee et al., 2000; Chen et al., 2003). This finding is supported by our RNAsequencing data that show robust expression of IMPG1 and IMPG2 in wild-type retinas (Murphy et al., 2016). In comparison, in the retinas that lack photoreceptor cells [AIPL1 $^{-1-}$ (aryl-hydrocarbon-interacting protein-like 1)], the expressions of IMPG1 and IMPG2 were negligible [IMPG1, 1425 (WT) vs $5\left(\mathrm{AIPL1}^{-l-}\right)$; IMPG2, 567 (WT) vs $7\left(\mathrm{AIPL1}^{-1-}\right)$; expression data are in arbitrary units]. We observed distinct compartmentalization of these molecules in the extracellular matrix. IMPG2 remains at the photoreceptor IS distal region. In contrast, IMPG1 was found along the OS region, up to the RPE (Fig. 1). In IMPG2 KO retinas, IMPG1 is mislocalized and mostly accumulated in the OS-RPE interface (Fig. 2). These findings imply that alteration at the IPM around the IS (IS-IPM) provokes deficits in the IPM around the OS (OSIPM), suggesting that the IS-IPM is involved in the generation of the OS-IPM (Fig. 8).

Lectins are proteins that bind specific carbohydrates. Two commonly used lectins to identify cone and rod photoreceptorspecific matrix are PNA and WGA, respectively (Hollyfield et al., 1990a,b; Johnson and Hageman, 1991; Tien et al., 1992). PNA recognizes galactosyl $(\beta-1,3) N$-acetyl galactosamine $(\mathrm{Gal} \beta 1$ $3 \mathrm{GalNAc}$ ) residues in glycoproteins (Cummings and Etzler, 2009), while WGA binds to the sialic acid commonly present around neurons (Monsigny et al., 1980; van der Want et al., 


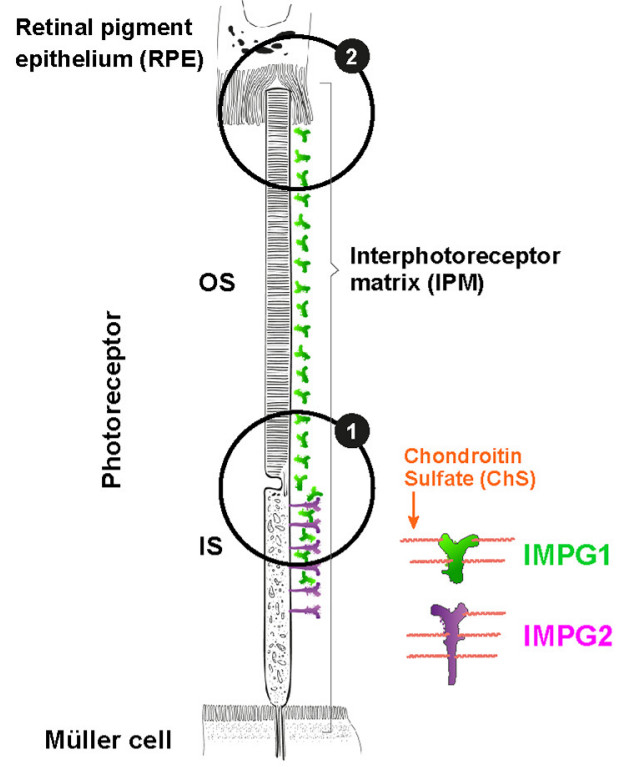

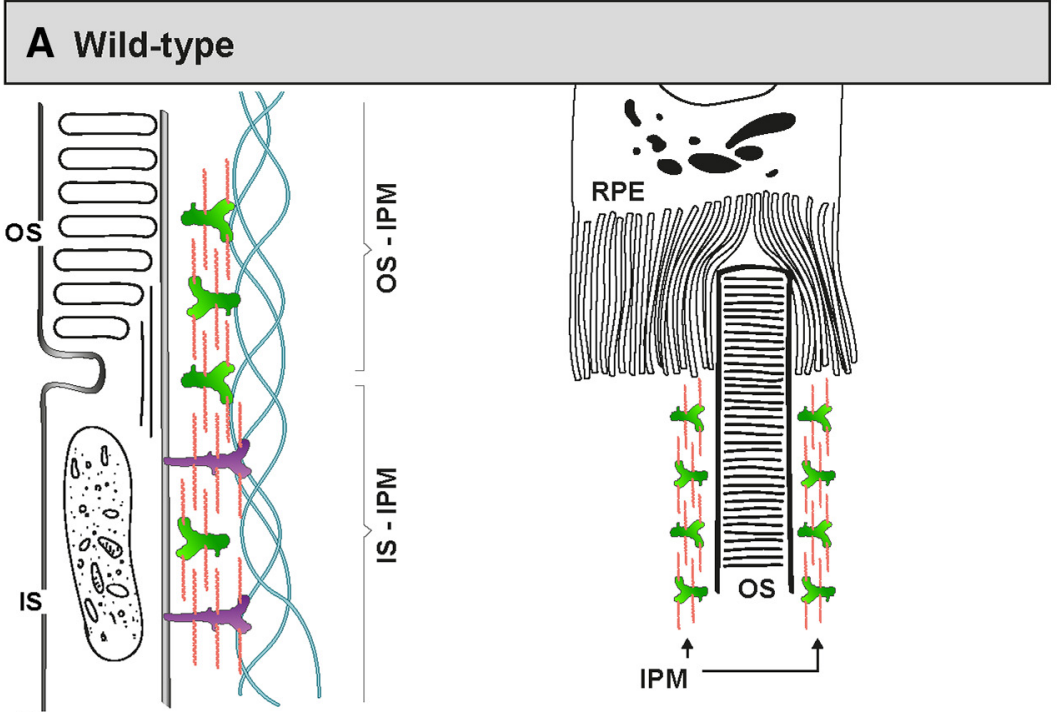

IMPG2 stabilizes the IS-IPM interaction promoting the distribution of IMPG1 along the OS-IPM axis.
2 As the RPE phagocytize the OS, IMPG1 is degraded by the RPE.

\section{B Deficit in IMPG2 proteoglycan}

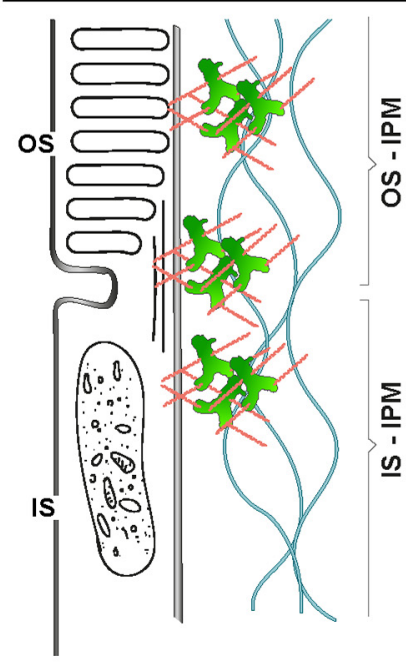

1 Absence of IMPG2 leads to disorganization of the IS-IPM and aggregation of IMPG 1.

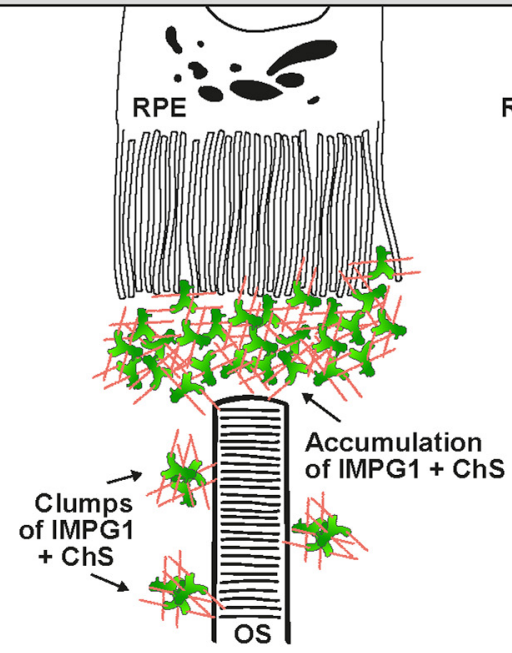

Aberrant accumulaOoOtion of IMPG1 between the RPE and OS.

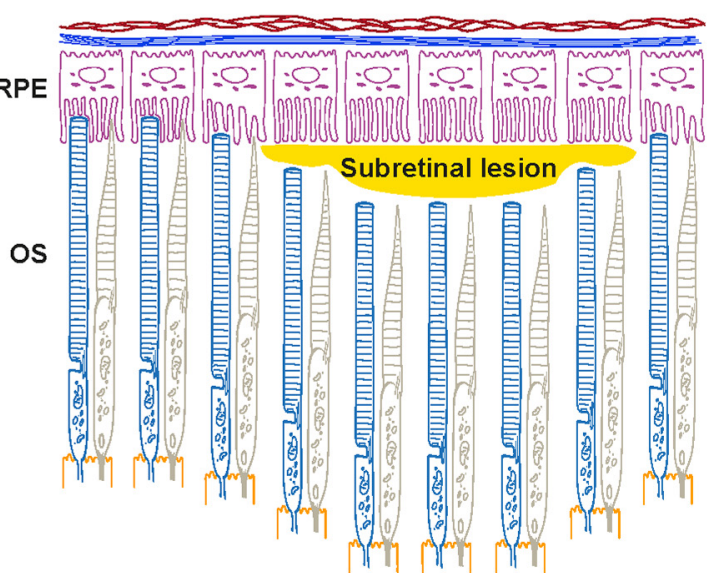

Over time, RPE fails to clear accumulated IMPG1 leading to subretinal lesions

Figure 8. Proposed mechanism of interaction between IMPG1 and IMPG2 in the generation of the IPM and the mechanism leading to vitelliform lesions in the absence of IMPG2. $\boldsymbol{A}$, Wild type showing IMPG2 (magenta) anchored to the photoreceptor IS cell membrane facing the IPM and exposing the ChS. IMPG2 proteoglycans interact with components of the IPM around the IS (IS-IPM) to arrange a specific IS-IPM spatial conformation that allows secreted IMPG1 to align in an ordered conformation. This ordered configuration of IMPG1 allows the protein to follow the OS maturation until it gets engulfed by the RPE. B, Lack of IMPG2 produces altered IS-IPM conformation with no guidance for the assembly of secreted IMPG1. Eventually, unorganized IMPG1 bundles together, forming clumps rich in ChS that interfere with the normal IPM clearance by the RPE. This forms a material that accumulates at the subretinal space and activates microglia. Over time, the RPE fails to remove the accumulated material leading to vitelliform lesions (arrow).

1997). Our IHC study showed that the PNA staining colocalizes with both IMPG1 and IMPG2 (Fig. 1), and follows the IMPG distribution in IMPG1 and IMPG2 KO mice (Fig. 2). In addition, staining for PNA was abolished in IMPG1-IMPG2 double KO (Fig. 6). These findings agree with previous studies that showed that PNA-lectin binds to IMPG1 and IMPG2 in the IPM. (Acharya et al., 2000; Hollyfield et al., 2001). Interestingly, although both rod and cone photoreceptors produce IMPG1 and IMPG2, PNA is used as a cone-matrix marker across multiple species. We speculate that the reason for the lack of IMPG1/ IMPG2 recognition of rod photoreceptor matrix is due to epitope masking of terminal sugar by sialyl residues. This is supported by studies showing that the removal of sialic acid by treatment eliminated the preferential binding of PNA to the cone IPM (Uehara, 1993; Uehara et al., 1995). In contrast, the staining pattern for WGA that recognizes sialic acid was unaltered in our animal models, suggesting a robust presence of sialylated proteins in the IPM. 
ChS is a primary component of numerous ECM biological tissues (Mikami and Kitagawa, 2013). The ChSs are linear polysaccharide chains composed of repeats of disaccharide units of glucuronic acid and $N$-acetylgalactosamine. The nomenclature of $\mathrm{ChS}$ is based on the position of sulfur groups substituted on the ChS backbone. There are three different types of ChS sulfation in the human retina, unsulfated, 4-sulfated and 6-sulfated ChS (Clark et al., 2011). However, in the mouse retina, 6-sulfated and weak 4-sulfated ChS were described (Hollyfield et al., 1999). Our findings show that ChS follows the anomalous IMPG localization pattern in single IMPG KOs. In addition, we observed a substantial reduction of ChS staining in the IPM of IMPG1IMPG2 double KO (Fig. 7), suggesting that IMPG proteoglycans are the primary source of 6-sulfated ChS at the IPM.

Our results show that IMPG proteoglycans are not essential for the development of functional rods and cones, indicating that these proteoglycans are not key players for OS development and maturation in mice (Figs. 2, 6). Surprisingly, IMPG1-IMPG2 double KO mice showed faint ChS staining at the IS, leaving the OS unstained (Fig. 7). We hypothesize that the absence of these normal constituents of the IPM initiates compensatory mechanisms that help to stabilize the IPM in the double $\mathrm{KO}$ mice. It is likely that the expression of some other ChS proteoglycan is upregulated to compensate for the loss of IMPGs. The exact mechanism behind this phenomenon is currently under investigation.

Humans with missense or nonsense mutations on IMPG2 are linked to two visual disorders, retinitis pigmentosa (RP) and AFVD. RP linked to IMPG mutations is characterized by early macular abnormalities (Bandah-Rozenfeld et al., 2010; van Huet et al., 2014). On the other hand, AFVD is characterized by round or oval, yellow, subretinal lesions forming an egg yolk-like vitelliform macular lesion associated with a relatively normal ERG (Hamel, 2014; Meunier et al., 2014; Alten and Eter, 2015; Chowers et al., 2015; Sivaprasad et al., 2016; Wilde et al., 2016; Brandl et al., 2017; Guziewicz et al., 2017; Khan and Al Teneiji, 2019). The reason behind the variability in the symptoms observed in humans is not clear. Our work shows that deficiency in IMPG2 proteoglycan in mouse models produces lesions like those found in humans with IMPG mutations. Humans with mutations in either IMPG protein develop subretinal lesions in areas with a high concentration of cones (Manes et al., 2013; Meunier et al., 2014; Brandl et al., 2017). We speculate that conespecific glycosylation due to IMPG is necessary for the stability of the cone-IPM. In this sense, the lack of macula in our animal model represents a limitation.

Young mice lacking IMPG2 at postnatal day 45 (P45) show early accumulation of material rich in IMPG1 and ChS between the OS and RPE, and in microglia cells migrating to the photoreceptor layers (Figs. 2, 3, 6G, 7). As the animals age, IMPG2 KO mice ( 5 months) develop subretinal lesions and a reduction of the ERG response (8 months; Figs. 4, 5). Animals lacking IMPG1 or both IMPG1 and IMPG2 do not show protein mislocalization or pathologic signs seen in IMPG2 KO mice, suggesting that the impairment of IMPG1 to integrate into the IPM and eventual accumulation in the subretinal space is the main cause of subretinal lesion formation and vision loss in IMPG2 KO mice.

The subretinal vitelliform lesions are also found in patients with truncated or dysfunctional IMPG1 proteins due to missense mutation of the IMPG1 gene (Manes et al., 2013; Meunier et al., 2014; Brandl et al., 2017). The findings from this work suggest that the abnormal IMPG1 proteins may not assemble properly in the IS-IPM and accumulate in the subretinal space, similar to our observation in the IMPG2 KO animal model.
Lesions in the CNS lead to an increase in the synthesis and deposition of ChS proteoglycans helping to form a glial seal; this phenomenon is also known to occur in retinal degenerative diseases (Singh et al., 2014; Dyck and Karimi-Abdolrezaee, 2015). IMPG proteins are highly glycosylated. The excessive accumulation of IMPG1 covalently bound to ChS at the RPE-OS boundary can form something akin to glial seal that over time may overwhelm the RPE uptake capacity, leading to the formation of subretinal lesions and visual impairment (Fig. 8).

Closer inspection of the subretinal lesions found in our IMPG2 KO mice reveals a dome-shaped mass that detaches the retina and occupies the subretinal space. These lesions contain IMPG1, stain with WGA, and present with a high concentration of microglia (Fig. 4). The photoreceptor neurons around the lesions show signs of damage, as indicated by the migration of microglia, reactive gliosis, and a reduction of the nuclear cell layer. Subretinal lesions in humans can be found as dome shaped (vitelliform lesions) or in a reticular pattern (SDDs), and both can be found in patients with IMPG mutations (Meunier et al., 2014). The SDDs are highly associated with the development of AMD (Boddu et al., 2014; Chowers et al., 2015; Sivaprasad et al., 2016). Based on these findings, we speculate that IMPG proteoglycans play an important role in the photoreceptor aging process.

In summary, our findings demonstrate that the aberrant localization of IPM proteoglycan is involved in the development of subretinal lesions. Based on our current findings, we propose that the accumulation of IMPG1 proteoglycan in the retina lacking IMPG2 might over time lead to RPE dysfunction (Fig. 8). Overall, our work suggests the existence of a dynamic, interconnected, and robust IPM that supports the RPE-photoreceptor microenvironment. The insights provided from this study are essential in deciphering the importance of the matrix in degenerative photoreceptor diseases and in the identification of novel therapies to treat matrix-associated blindness.

\section{References}

Acharya S, Foletta VC, Lee JW, Rayborn ME, Rodriguez IR, Young WS 3rd, Hollyfield JG (2000) SPACRCAN, a novel human interphotoreceptor matrix hyaluronan-binding proteoglycan synthesized by photoreceptors and pinealocytes. J Biol Chem 275:6945-6955.

Alten F, Eter N (2015) Current knowledge on reticular pseudodrusen in agerelated macular degeneration. Br J Ophthalmol 99:717-722.

Arranz AM, Perkins KL, Irie F, Lewis DP, Hrabe J, Xiao F, Itano N, Kimata K, Hrabetova S, Yamaguchi Y (2014) Hyaluronan deficiency due to Has3 knock-out causes altered neuronal activity and seizures via reduction in brain extracellular space. J Neurosci 34:6164-6176.

Bandah-Rozenfeld D, Collin RWJ, Banin E, Ingeborgh van den Born L, Coene KLM, Siemiatkowska AM, Zelinger L, Khan MI, Lefeber DJ, Erdinest I, Testa F, Simonelli F, Voesenek K, Blokland EAW, Strom TM, Klaver CCW, Qamar R, Banfi S, Cremers FPM, Sharon D, et al. (2010) Mutations in IMPG2, encoding interphotoreceptor matrix proteoglycan 2, cause autosomal-recessive retinitis pigmentosa. Am J Hum Genet 87:199-208.

Berardi N, Pizzorusso T, Maffei L (2004) Extracellular matrix and visual cortical plasticity: freeing the synapse. Neuron 44:905-908.

Boddu S, Lee MD, Marsiglia M, Marmor M, Freund KB, Smith RT (2014) Risk factors associated with reticular pseudodrusen versus large soft drusen. Am J Ophthalmol 157:985-993.e2.

Brandl C, Schulz H, Charbel Issa P, Birtel J, Bergholz R, Lange C, Dahlke C, Zobor D, Weber B, Stöhr H (2017) Mutations in the genes for interphotoreceptor matrix proteoglycans, IMPG1 and IMPG2, in patients with vitelliform macular lesions. Genes (Basel) 8:170.

Caterson B (2012) Fell-Muir Lecture: chondroitin sulphate glycosaminoglycans: fun for some and confusion for others. Int J Exp Pathol 93:1-10. 
Caterson B, Christner JE, Baker JR, Couchman JR (1985) Production and characterization of monoclonal antibodies directed against connective tissue proteoglycans. Fed Proc 44:386-393.

Chen Q, Lee JW, Nishiyama K, Shadrach KG, Rayborn ME, Hollyfield JG (2003) SPACRCAN in the interphotoreceptor matrix of the mouse retina: molecular, developmental and promoter analysis. Exp Eye Res 76:1-14.

Chowers I, Tiosano L, Audo I, Grunin M, Boon CJ (2015) Adult-onset foveomacular vitelliform dystrophy: a fresh perspective. Prog Retin Eye Res 47:64-85.

Clark SJ, Keenan TD, Fielder HL, Collinson LJ, Holley RJ, Merry CL, van Kuppevelt TH, Day AJ, Bishop PN (2011) Mapping the differential distribution of glycosaminoglycans in the adult human retina, choroid, and sclera. Invest Ophthalmol Vis Sci 52:6511-6521.

Couchman JR, Caterson B, Christner JE, Baker JR (1984) Mapping by monoclonal antibody detection of glycosaminoglycans in connective tissues. Nature 307:650-652.

Cummings RD, Etzler ME (2009) Antibodies and lectins in glycan analysis. In: Essentials of glycobiology, Chap 45, (Varki A, Cummings RD, Esko JD, Freeze HH, Stanley P, Bertozzi CR, Hart GW, Etzler ME, eds). Cold Spring Harbor, NY: Cold Spring Harbor Laboratory.

Dilan TL, Moye AR, Salido EM, Saravanan T, Kolandaivelu S, Goldberg AFX, Ramamurthy V (2019) ARL13B, a Joubert syndrome-associated protein, is critical for retinogenesis and elaboration of mouse photoreceptor outer segments. J Neurosci 39:1347-1364.

Dyck SM, Karimi-Abdolrezaee S (2015) Chondroitin sulfate proteoglycans: key modulators in the developing and pathologic central nervous system. Exp Neurol 269:169-187.

Felemban M, Dorgau B, Hunt NC, Hallam D, Zerti D, Bauer R, Ding Y, Collin J, Steel D, Krasnogor N, Al-Aama J, Lindsay S, Mellough C, Lako M (2018) Extracellular matrix component expression in human pluripotent stem cell-derived retinal organoids recapitulates retinogenesis in vivo and reveals an important role for IMPG1 and CD44 in the development of photoreceptors and interphotoreceptor matrix. Acta Biomater 74:207-221

Foletta VC, Nishiyama K, Rayborn ME, Shadrach KG, Young WS 3rd, Hollyfield JG (2001) SPACRCAN in the developing retina and pineal gland of the rat: spatial and temporal pattern of gene expression and protein synthesis. J Comp Neurol 435:354-363.

Garlipp MA, Nowak KR, Gonzalez-Fernandez F (2012) Cone outer segment extracellular matrix as binding domain for interphotoreceptor retinoidbinding protein. J Comp Neurol 520:756-769.

Guziewicz KE, Sinha D, Gómez NM, Zorych K, Dutrow EV, Dhingra A, Mullins RF, Stone EM, Gamm DM, Boesze-Battaglia K, Aguirre GD (2017) Bestrophinopathy: an RPE-photoreceptor interface disease. Prog Retin Eye Res 58:70-88.

Hamel CP (2014) Gene discovery and prevalence in inherited retinal dystrophies. C R Biol 337:160-166.

Hauck SM, Schoeffmann S, Deeg CA, Gloeckner CJ, Swiatek-de Lange M, Ueffing M (2005) Proteomic analysis of the porcine interphotoreceptor matrix. Proteomics 5:3623-3636.

Hensch TK (2005) Critical period plasticity in local cortical circuits. Nat Rev Neurosci 6:877-888.

Hollyfield JG, Varner HH, Rayborn ME (1990a) Regional variation within the interphotoreceptor matrix from fovea to the retinal periphery. Eye (Lond) 4:333-339.

Hollyfield JG, Rayborn ME, Landers RA, Myers KM (1990b) Insoluble interphotoreceptor matrix domains surround rod photoreceptors in the human retina. Exp Eye Res 51:107-110.

Hollyfield JG, Rayborn ME, Tammi M, Tammi R (1998) Hyaluronan in the interphotoreceptor matrix of the eye: species differences in content, distribution, ligand binding and degradation. Exp Eye Res 66: 241-248.

Hollyfield JG, Rayborn ME, Midura RJ, Shadrach KG, Acharya S (1999) Chondroitin sulfate proteoglycan core proteins in the interphotoreceptor matrix: a comparative study using biochemical and immunohistochemical analysis. Exp Eye Res 69:311-322.

Hollyfield JG, Rayborn ME, Nishiyama K, Shadrach KG, Miyagi M, Crabb JW, Rodriguez IR (2001) Interphotoreceptor matrix in the fovea and peripheral retina of the primate Macaca mulatta: distribution and glycoforms of SPACR and SPACRCAN. Exp Eye Res 72:49-61.
Ishikawa M, Sawada Y, Yoshitomi T (2015) Structure and function of the interphotoreceptor matrix surrounding retinal photoreceptor cells. Exp Eye Res 133:3-18.

Johnson LV, Hageman GS (1991) Structural and compositional analyses of isolated cone matrix sheaths. Invest Ophthalmol Vis Sci 32:19511957.

Keenan TD, Clark SJ, Unwin RD, Ridge LA, Day AJ, Bishop PN (2012) Mapping the differential distribution of proteoglycan core proteins in the adult human retina, choroid, and sclera. Invest Ophthalmol Vis Sci 53:7528-7538.

Khan AO, Al Teneiji AM (2019) Homozygous and heterozygous retinal phenotypes in families harbouring IMPG2 mutations. Ophthalmic Genet 40:247-251.

Kuehn MH, Hageman GS (1999a) Expression and characterization of the IPM 150 gene (IMPG1) product, a novel human photoreceptor cell-associated chondroitin-sulfate proteoglycan. Matrix Biol 18:509-518.

Kuehn MH, Hageman GS (1999b) Molecular characterization and genomic mapping of human IPM 200, a second member of a novel family of proteoglycans. Mol Cell Biol Res Commun 2:103-110.

Lazarus HS, Hageman GS (1992) Xyloside-induced disruption of interphotoreceptor matrix proteoglycans results in retinal detachment. Invest Ophthalmol Vis Sci 33:364-376.

Lee JW, Chen Q, Rayborn ME, Shadrach KG, Crabb JW, Rodriguez IR, Hollyfield JG (2000) SPACR in the interphotoreceptor matrix of the mouse retina: molecular, biochemical and immunohistochemical characterization. Exp Eye Res 71:341-352.

Manes G, Meunier I, Avila-Fernández A, Banfi S, Le Meur G, Zanlonghi X, Corton M, Simonelli F, Brabet P, Labesse G, Audo I, Mohand-Said S, Zeitz C, Sahel J-A, Weber M, Dollfus H, Dhaenens C-M, Allorge D, De Baere E, Koenekoop RK, et al. (2013) Mutations in IMPG1 cause vitelliform macular dystrophies. Am J Hum Genet 93:571-578.

Meunier I, Manes G, Bocquet B, Marquette V, Baudoin C, Puech B, Defoort-Dhellemmes S, Audo I, Verdet R, Arndt C, Zanlonghi X, Le Meur G, Dhaenens CM, Hamel CP (2014) Frequency and clinical pattern of vitelliform macular dystrophy caused by mutations of interphotoreceptor matrix IMPG1 and IMPG2 genes. Ophthalmology 121: 2406-2414.

Mikami T, Kitagawa H (2013) Biosynthesis and function of chondroitin sulfate. Biochim Biophys Acta 1830:4719-4733.

Monsigny M, Roche AC, Sene C, Maget-Dana R, Delmotte F (1980) Sugarlectin interactions: how does wheat-germ agglutinin bind sialoglycoconjugates? Eur J Biochem 104:147-153.

Moye AR, Singh R, Kimler VA, Dilan TL, Munezero D, Saravanan T, Goldberg AFX, Ramamurthy V (2018) ARL2BP, a protein linked to retinitis pigmentosa, is needed for normal photoreceptor cilia doublets and outer segment structure. Mol Biol Cell 29:1590-1598.

Murphy D, Cieply B, Carstens R, Ramamurthy V, Stoilov P (2016) The Musashi 1 controls the splicing of photoreceptor-specific exons in the vertebrate retina. PLoS Genet 12:e1006256.

Nicholson C, Hrabětová S (2017) Brain extracellular space: the final frontier of neuroscience. Biophys J 113:2133-2142.

Okunuki Y, Mukai R, Pearsall EA, Klokman G, Husain D, Park DH, Korobkina E, Weiner HL, Butovsky O, Ksander BR, Miller JW, Connor KM (2018) Microglia inhibit photoreceptor cell death and regulate immune cell infiltration in response to retinal detachment. Proc Natl Acad Sci U S A 115:E6264-E6273.

Singh RK, Kolandaivelu S, Ramamurthy V (2014) Early alteration of retinal neurons in Aipl1-/- animals. Invest Ophthalmol Vis Sci 55:3081-3092.

Sivaprasad S, Bird A, Nitiahpapand R, Nicholson L, Hykin P, Chatziralli I (2016) Perspectives on reticular pseudodrusen in age-related macular degeneration. Surv Ophthalmol 61:521-537.

Sorg BA, Berretta S, Blacktop JM, Fawcett JW, Kitagawa H, Kwok JC, Miquel M (2016) Casting a wide net: role of perineuronal nets in neural plasticity. J Neurosci 36:11459-11468.

Strauss O (2005) The retinal pigment epithelium in visual function. Physiol Rev 85:845-881.

Tien L, Rayborn ME, Hollyfield JG (1992) Characterization of the interphotoreceptor matrix surrounding rod photoreceptors in the human retina. Exp Eye Res 55:297-306.

Uehara F (1993) [Molecular cell glycobiology of the retina]. Nippon Ganka Gakkai Zasshi 97:1370-1393. 
Uehara F, Ozawa M, Sameshima M, Unoki K, Okubo A, Yanagita T, Sugata M, Iwakiri N, Maeda Y, Muramatsu T (1995) Differential expression of mRNA for alpha 2,3-sialyltransferase during development of rat retina. Jpn J Ophthalmol 39:248-253.

van der Want JJ, Klooster J, Cardozo BN, de Weerd H, Liem RS (1997) Tract-tracing in the nervous system of vertebrates using horseradish peroxidase and its conjugates: tracers, chromogens and stabilization for light and electron microscopy. Brain Res Brain Res Protoc 1:269-279.

van Huet RA, Collin RW, Siemiatkowska AM, Klaver CC, Hoyng CB, Simonelli F, Khan MI, Qamar R, Banin E, Cremers FP, Theelen T, den Hollander AI, van den Born LI, Klevering BJ (2014) IMPG2-associated retinitis pigmentosa displays relatively early macular involvement. Invest Ophthalmol Vis Sci 55:3939-3953.
Wen TH, Binder DK, Ethell IM, Razak KA (2018) The perineuronal "safety" net? perineuronal net abnormalities in neurological disorders. Front Mol Neurosci 11:270.

Wilde C, Lakshmanan A, Patel M, Morales MU, Dhar-Munshi S, Amoaku WM (2016) Prevalence of reticular pseudodrusen in newly presenting adult onset foveomacular vitelliform dystrophy. Eye (Lond) 30:817-824.

Xie L, Kang $\mathrm{H}, \mathrm{Xu}$ Q, Chen MJ, Liao Y, Thiyagarajan M, O'Donnell J, Christensen DJ, Nicholson C, Iliff JJ, Takano T, Deane R, Nedergaard M (2013) Sleep drives metabolite clearance from the adult brain. Science 342:373-377.

Zweifel SA, Spaide RF, Yannuzzi LA (2011) Acquired vitelliform detachment in patients with subretinal drusenoid deposits (reticular pseudodrusen). Retina 31:229-234. 